\title{
A New Hybrid Adaptive Mesh Algorithm Based on Voronoi Tessellations and Equi- distribution Principle: Algorithms and Numerical Experiments
}

\author{
Shaolin Mao ${ }^{1}$ \\ Department of Mechanical Engineering, The University of Texas at El Paso, TX 79968, USA
}

\begin{abstract}
A new hybrid h-adaptive algorithm is design to optimize unstructured polygonal meshes in $2 \mathrm{D}$ based on Voronoi tessellations and its topological dual, Delaunay triangulations. The algorithm consists of several unique features: first, dynamic change of mesh topology (reconnection of nodes) is realized through refinement and coarsening iterations with specific feature (i.e. Voronoi diagram); second, two moving mesh strategies are included, one is the weighted global smoothing for mesh regulation which is based on centroidal Voronoi tessellations (CVTs), the other is a local smoothing for the purpose of speed-up of the adaptive iterations; third, a new marking strategy with double threshold values guarantee the convergence of adaptive iterations. The advantage of the hybrid algorithm is that the goal mesh resolution can converge to an arbitrarily small number; it asymptotically converges to a straight line in $2 \mathrm{D}$ (i.e., the volume equi-distribution error approaches zero). The $h$-adaptation is referred to as asynchronous process, if the processes of coarsening and refinement are connected through several global mesh regulation steps. Otherwise the hybrid adaptive process is synchronous. This will provide options to meet special requirement such as smooth transition of physical fields. The numerical results show that the numerical errors are indeed well distributed in an averaged sense in the domain, where the mesh sizes are optimal, and nodes of the mesh smoothly follow the underlying geometry or physics. The new hybrid algorithm demonstrates its potential for effectiveness, efficiency and robustness to handle generation and optimization of polygonal meshes for extreme scenarios with a wide spectrum of length scales.
\end{abstract}

\footnotetext{
${ }^{1}$ (LANL Technical Report No.: LA-UR-12-21651). Tel: +1 915747 5830, E-mail: smao@utep.edu, slm wvu@yahoo.com
} 
Keywords: Hybrid adaptive, h-adaptive, Voronoi tessellation, Delaunay triangulation, equidistribution, mesh regulation, mesh optimization

\section{Introduction}

The accuracy of numerical solutions of partial differential equations is highly dependent on the quality of the mesh, in particular, with the underlying physics of complicated structure, interaction between fluids and solids, small length scales of shock waves, singularities and problems with free surfaces and moving boundaries (e.g., Shyy et al. 1996; ferziger and Peric 2000; Deville et al. 2002; Prosperetti and Tryggvason 2007; Feng et al. 2012; Mao et al. 2007; 2008; 2010). The approach of dynamic adaptive mesh can significantly improve the accuracy and efficiency of computation. The application of adaptation is attractive because it permits mesh resolution to be focused on specific region of computation domain of the solution and it leads to substantial saving of computational time. There are two groups of widely-used adaptive mesh, the local adaptive mesh refinement methods (also known as the $h$-adaptive) and the moving mesh methods (also known as the $r$-adaptive). There have extensive studies on the moving mesh methods in past decades (e.g., Brackbill and Saltzmann 1982; Thompson et al. 1985; Knupp and Steinberg 1994; Liseikin 1999; Delzanno et al. 2008; 2011; Chacon et al. 2011; Budd and Williams 2006; 2009; Huang 2005; Li et al. 1998; Lapenta 2003). The advantages of the moving mesh methods are their simple data structure, smooth results and good for time-dependent problems. The drawback of moving methods resides in their limited capability for resolution of local flow features with great distortion of the meshes. On the other hand, the $h$-adaptive algorithms have the remarkable features such as simplicity of concept, robustness, effectiveness of implementation and it can avoid the possible mesh distortion and mesh tangling problems 
even though the data structure of the $h$-adaptive methods are relatively complicated compared to the moving mesh methods.

The motivation of this study is to investigate a new hybrid $h$-adaptive algorithm with the capability of variable mesh topology and optimal meshes in terms of equi-distribution of mesh resolutions. The change of the mesh topology, i.e. the reconnection of cell nodes, can lead to an effective way to avoid mesh distortion and entangling phenomena, a big challenging problem that plagued moving mesh methods in complex flow modeling (Brackbill and Saltzmann 1982; Winslow 1981). A possible direct application of the novel algorithm is the arbitrary LagrangianEulerian (ALE) methods (Loubere et al. 2010; Norris et al. 2010). In past decades Voronoi diagram/tessellation techniques have been widely used in computer graphics and network applications (Okabe et al. 2000). It becomes a powerful tool in modeling of multiphase multimaterial flows, atmospheric and climate, and extreme material problems, among others (Kim et al. 2005; Khamayseh and Hansen 2008; Lipnikov and Shashkov 2006; Du and Gunzburger 2010; Evazi and Mahani 2010; Springel 2009; Weller et al. 2009). Especially the socalled centroidal Voronoi tessellations (CVTs) demonstrate its simplicity of implementation and effectiveness of mesh generation (Du et al. 1999; Du and Gunzburger 2002).

The essence of the novel methodology is to effectively build a way to reconnect the nodes of a mesh, for instance, an initial triangle mesh could evolve to quadrilateral or other polygonal mesh in order to serve the goal of optimal distribution of the mesh resolution. The process of generating new mesh is exactly a procedure of polygonal mesh optimization. Here there are two major questions that we need to answer, what type of metrics to use to evaluate the mesh quality and how to implement it. For the first question, a new metrics based on Gersho's conjecture (1979) is used to evaluate mesh quality at each node, and the mesh resolution will be adjusted 
through the equi-distribution principle. For the second question, update of each polygonal mesh is carried out using Voronoi tessellations and its topological dual, the Delaunay triangulation. There are several ways to directly generate Voronoi tessellations, for instance, the incremental method, the divide-and-conquer method, and the plane sweep method. The Voronoi generation technique used in this study will be summarized in Section 2, but the detailed discussion of Voronoi tessellations algorithm can be found in Okabe et al. (2000) and Shewchuk (1999).

We introduce a novel approach which inherits the structural simplicity and smooth transition of the $r$-adaptation and the effectiveness and robustness of the $h$-adaptation. Hereafter the novel approach is referred to as the hybrid $h$-adaptation. Although various numerical methods have been implemented using the $h$-adaptive methodology for unstructured mesh such as Delaunay grid control, advancing front grid control, and the quadtree algorithm (Thompson et al. 1985; 1999; Tabarrael and Sukumar 2007). The hybrid $h$-adaptive algorithm offers significant advantage over conventional methods for the mesh generation and optimization, especially targets in multi-scale modeling and simulation. First, it provides a well balance between the local mesh smoothing and the global mesh regulation for a given distribution requirement (e.g., a monitor function). The local features, either physical or geometrical, can be perfectly resolved without much overhead of the overall computation load. Second, the hybrid algorithm brings the flexibility of so-called asynchronous process into the implementation of the coarsening and refinement iterations. Depending on the transition rate of mesh sizes, several mesh global regulation ( $r$-adaptive) sweeps are applied to connect the separate coarsening and refinement iterations (referred to as asynchronous process, otherwise synchronous). This could avoid any possible counter-flow phenomenon during the adaptation iterations (i.e., coarsening-refinementcoarsening loop in the same region, which resembles oscillation of iterations). Third, the hybrid 
algorithm offers better control of mesh generation and optimization nearby boundary region by ways of generation of Voronoi tessellations. Because the evaluation of mesh quality is based on vertices of each Voronoi cell (i.e., the circumcenter of its dual Delaunay triangle), it avoids the solution of complex Jacobian system between the mapping from the old mesh to the new one. Finally, an interesting result from the hybrid algorithm is that the goal mesh resolution can converge to an arbitrarily small value to meet the spatial discretization requirement.

The remainder of this paper is organized as follows. Section 2 describes the complete hybrid $h$-adaptive algorithm. Metric study and efficacy test of the hybrid algorithm will be provided in Section 3. Finally is the conclusion about the novel algorithm. In a separate but related paper, Part II: Applications, finite volume method combined with the novel algorithm will be demonstrated including more numerical experiments and numerical error discussion.

\section{The Hybrid Adaptive Algorithm}

In this section we illustrate the steps of the hybrid $h$-adaptive algorithm which is based on the Voronoi tessellation and its topological dual, the Delaunay triangulation. There are many robust techniques to generate Voronoi tessellations and Delaunay triangles (e.g., Chapter 4 in Okabe et al. 2000; Shewchuk 1999). Once the Voronoi tessellation is established, we calculate the area, the centers of cells and corresponding mesh resolution. To this end, we introduce a mesh quality metric and explain a novel marking strategy. As soon as the region to be refined or coarsened is determined we conduct the refinement and coarsening iterations. Note that the refinement and coarsening can be either synchronous or asynchronous. Two smoothing strategies are introduced for different purposes, one for global mesh regulation and the other for speedingup the convergence of adaptive iterations (hereafter called as the local smoothing). Finally, the optimal shape of Voronoi cells is evaluated by the equi-distribution principle for a given monitor 
function or numerical error estimator of a PDE solution. Even though the proposed algorithm is focusing on convex geometry in $2 \mathrm{D}$, it can be extended to 3D geometries with different metrics of mesh resolutions.

Voronoi tessellations and generators. We introduce the definition of generator and Voronoi tessellation/diagram (hereafter referenced to as cells). Given an open bounded domain $\Omega \in R^{2}$ and a set of distinct points $\left\{z_{i}\right\}_{i=1}^{n} \in \Omega$. For each point $z_{i}$ define the corresponding Voroni cell $V_{i}$ by

$V_{i}=\left\{z \in \Omega \mid\left\|z-z_{i}\right\|<\left\|z-z_{j}\right\|\right.$ for $j=1, \cdots, n$ and $\left.j \neq i\right\}$

Based on above definition, the $\left\{V_{i}\right\}_{i=1}^{n}$ is a tessellation of $\Omega$. A point $z_{i}$ is called as a generator of its corresponding tessellation. It is well known that the dual tessellation to a Voronoi tessellation is a so-called Delaunay dual triangulation (Okabe et al. 2000). There are effective and robust techniques available to generate Voronoi tessellations.

\subsection{Generation of initial polygonal meshes}

For the sake of completeness, the generation of initial polygonal mesh is summarized here. One approach is to directly generate Voronoi tessellations. The incremental method and the divide-and-conquer technique are two representatives. For instance, the divide-and-conquer method recursively divides the original problem into several simple sub-problems of almost equal size, and obtains the solution of original problem by merging the solutions of the subproblems. Given the number of generators in the domain, $i=1, \cdots, n$, the simplest case is $n \leq 3$ which can generate the Voronoi tessellations by its definition directly. If $n>3$ we can recursively divide the domain, for instance, the left side and right side with $n / 2$ number of generators on each side. The critical step in the divide-and-conquer technique is the merging of two neighbor tessellations into a new one. For details about the merge algorithm, the reader is 
referred to Okabe et al. (2000). There exist different types of divide-and-conquer methods with slightly different construction time on average such as $O(n)$ to $O(n \log n)$.

The second approach is to generate Delaunay triangles first, its topological dual, the Voronoi cells will be created then (Shewchuk 1999). The common used algorithms to constructing Delauny triangulations are incremental insertion by Bowyer and Watson, the divideand-conquer, and the sweepline technique. The second approach is a standard practice that was applied in this study except a Dirichlet type dmain needed here.

\subsection{The $h$-adaptive algorithm}

The $h$-adaptive algorithm consists of two independent components, the coarsening process and the refinement process. Both of them are conducted based on the volume equi-distribution of a monitor function (distribution function). The equi-distribution is an important principle for reduction of the numerical errors of PDE solutions. It has been shown that the equi-distribution of the local error leads to minimization of the global error (Lapenta 2003). In one dimension, the equi-distribution principle leads to error equi-distribution and a rigorous minimization procedure on each node (Huang 2005). However, in two dimensions, the equi-distribution of a monitor function optimizes the value of the monitor function on each vertex of polygonal cells but the minimization procedure is not unique (Huang 2003).

Mesh resolution. For bounded and strictly positive monitor function, the size (i.e., volume in $3 \mathrm{D}$ ) of a Voronoi cell is inversely proportional to the one-third power of the underlying density at the middle point of the Voronoi cell (Gersho 1979). This conjecture can be used to construct the metric for the equi-distribution principle which can lead to a unique optimized mesh in terms of an averaged volume equi-distribution error. The following idea has been applied to imaging and data process problems before (e.g., Cappellari and Copin 2003; 
Emelianenko 2010). Given a monitor function $f(x, y)$ which can be a weighted distribution or $a$ posteriori numerical error of PDE, $A$ is the two-dimensional domain $\Omega, N$ is the number of generators in the domain, $R$ is the goal resolution coefficient of the final optimal polygonal mesh. The equi-distribution condition reads

$\frac{\sqrt{f\left(x_{1}, y_{1}\right)}+\sqrt{f\left(x_{2}, y_{2}\right)}+\sqrt{f\left(x_{3}, y_{3}\right)}}{3} A_{123}=\frac{1}{R} \int_{\Omega} \sqrt{f(x, y)} d A$

where $A_{123}$ is the area of the Delaunay triangle whose vertices are "1", "2" and " 3 ", which are the corresponding generators of the three neighbor topological dual Voronoi cells (Figure 1). The three vertices of the Delaunay triangle are denoted by open circles while the vertices of each Voronoi cell are marked by filled solid circles.

\section{O Generators \\ - Voronoi edges \\ - Delaunay edges}

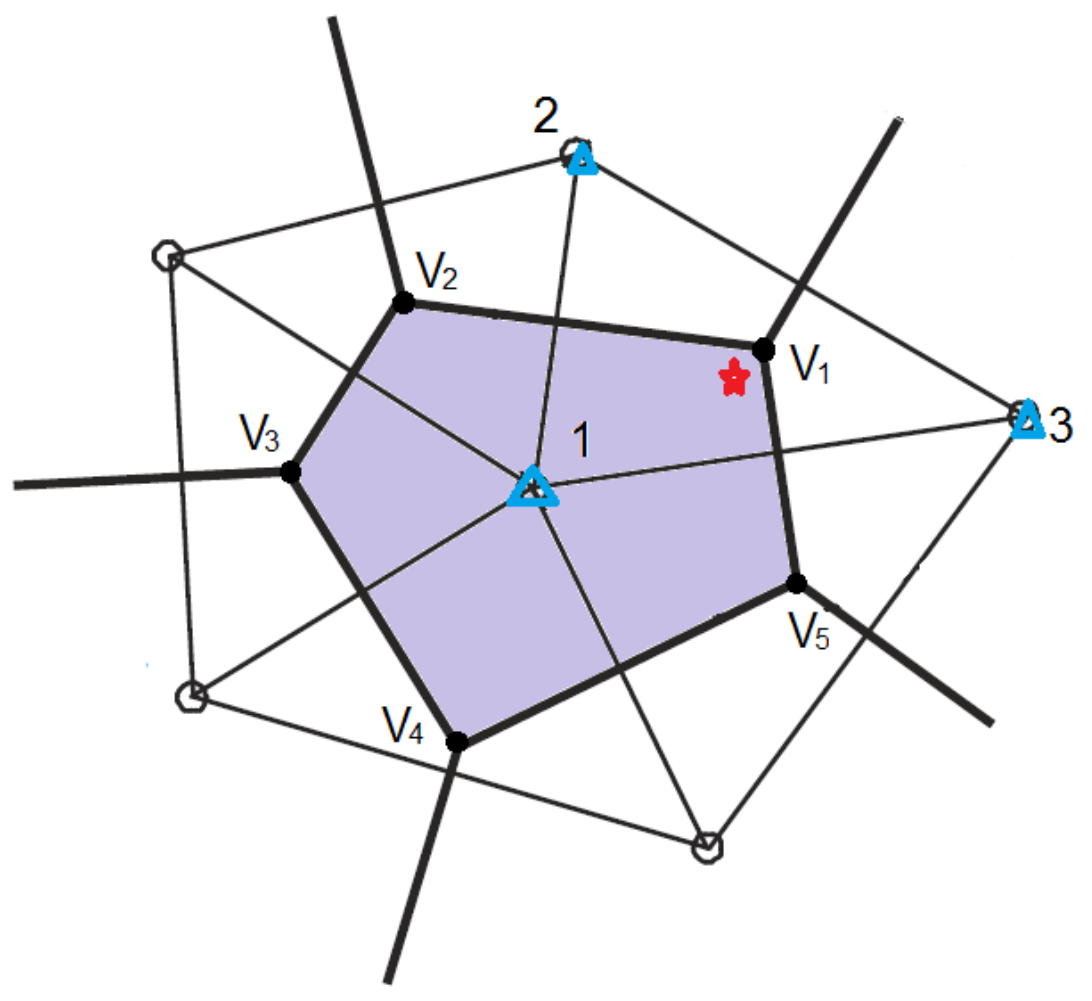

Figure 1: Voronoi cells and its topological dual Delaunay triangles in 2D domain. The vertices of the Voronoi cells are marked by filled black circles while the open circles stand for the vertices of the Delaunay triangles. The small red star denotes the location where a new generator will be added to the domain. 
If in Eqn. (2) the left hand side and the right hand side approximately equal to each other at each node of the polygonal mesh, we claim that the mesh is optimal with respect to the minimal value of monitor function (e.g., minimized numerical error). Mostly this condition does not hold for a given initially uniform or arbitrary mesh. For this reason, we define the right hand side of Eqn. (2) as the normalized goal mesh resolution

$M=\frac{1}{R} \int_{\Omega} \sqrt{f(x, y)} d A$

We refer to the left hand side as the real mesh resolution at the current node of the mesh.

Eqn. (2) for equi-distribution of mesh quality is a cornerstone for the algorithm below. The justification can be clearer if we rewrite it in a much familiar way about the idea of equidistribution (Delzanno et al. 2008; Budd and Williams 2009)

$\frac{\int_{\Omega_{L}} \sqrt{f(x, y)} d A}{\frac{1}{R} \int_{\Omega} \sqrt{f(x, y)} d A}=\frac{\int_{\mathcal{C}} d \mathcal{A}}{\int_{\Omega_{\mathcal{C}}} d \mathcal{A}}$

The right hand side of above equation is nothing else but a reference mesh (usually uniform mesh) while the left hand side is the physical mesh (i.e. Voronoi tessellations). $\mathcal{C}$ is a set of local nodes, $\Omega_{\mathcal{C}}$ is the domain for a reference mesh. $\Omega_{L}$ is the corresponding image set of $\mathcal{C}$. The only difference from conventional approach is the application of $\sqrt{f(x, y)}$ instead of $f(x, y)$ (e.g., scalar Stieltijes measure). In order to be consistent with equi-distribution idea, $f(x, y)$ is called the monitor function or density function. If we limit the set of nodes (generators) to 3 , the easiest way, to discretize the integral $\int_{\Omega_{L}} \sqrt{f(x, y)} d A$, Eqn. (2) will be recovered.

Coarsening process. In order to reach the goal of equi-distribution of the mesh resolution, the following coarsening or refinement iterations will be conducted until the real mesh resolution each node approximately equal to the goal mesh resolution $\mathrm{M}$ with a tolerance of $\varepsilon$. A novel 
threshold-based marking strategy is proposed here, which is different from other available threshold-based algorithm (e.g. Dorfler 1996), to serve this goal. Section 2.2 will explain the marking strategy in detail. For coarsening sweeps, we loop each node in the domain $\Omega$, calculate individual distribution (i.e. the real mesh resolution), then construct a subset of the whole Voronoi tessellations such that

$\frac{\sqrt{f\left(x_{1}, y_{1}\right)}+\sqrt{f\left(x_{2}, y_{2}\right)}+\sqrt{f\left(x_{3}, y_{3}\right)}}{3} A_{123}<\varphi_{c} \frac{1}{R} \int_{\Omega} \sqrt{f(x, y)} d A$

We then mark all generators in the subset. All marked generators in current mesh $M^{n}$ will be eliminated in the new mesh $M^{n+1}$ which is the direct result after conducting this coarsening step. Here the subscript indices "1", "2" and " 3 " have the same meaning as before (Figure 1). The threshold parameter $\varphi_{c}$ is a prescribed value to determine the scope or bandwidth of Voronoi cells to be coarsened. The right hand side of the above inequality stands for the lower bound of the goal mesh resolution

$\underline{M}=\varphi_{c} \frac{1}{R} \int_{\Omega} \sqrt{f(x, y)} d A$

It is important to note that the threshold parameter is not a universal value but problemdependent. For instance, the following numerical experiments choose $\varphi_{c}=0.875$ and remain a constant during coarsening sweeps for the sake of simplicity. In fact, this parameter can be prescribed. Figure 1 shows the marking strategy in which the repeated generators will be marked only once (the small blue triangles) as well as a new generator (the small red star). During the generation step, the position of the new generator is determined by

$x^{*}=\frac{x_{1}+x_{2}+x_{3}}{3}, \quad y^{*}=\frac{y_{1}+y_{2}+y_{3}}{3}$

with $\left(x_{1}, y_{1}\right),\left(x_{2}, y_{2}\right),\left(x_{3}, y_{3}\right)$ being the three vertices of the dual Delaunay triangle. 
Skip Process. The above coarsening sweep is a simple and effective process, mostly 3-5 iterations, to reach locally optimal distribution of the number of generators for the examples presented in this paper. The only exception is the scenario in which the number of generators to be added is very close to the number of generators to be eliminated. For instance, if one region needs coarsening and its neighbor region needs refinement, there would be a dilemma which leads to a very slow convergence of coarsening sweeps. Figure 2 shows an example about convergence problem between coarsening and refinement due to the lack of a buffer zone between coarsening and refinement.

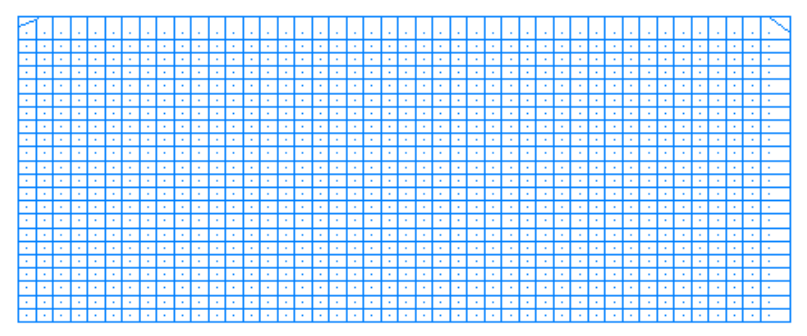

(a)

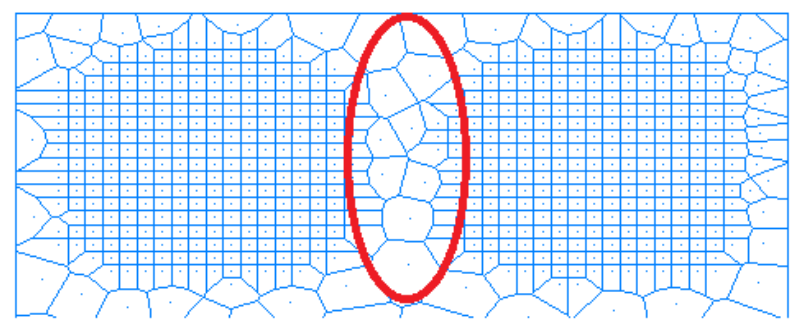

(b)

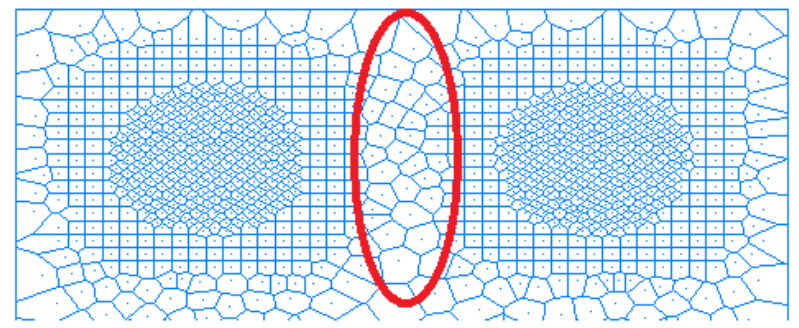

(c)

Figure 2: Convergence problem due to lack of a buffer zone between coarsening and refinement process. (a) The initial uniform mesh; (b) coarsening sweeps, the middle region was overcoarsened; (c) the same region was refined and would be coarsened again.

In order to overcome the difficulty associated with slow iteration convergence in Figure 2, a so-called "skip algorithm" has been considered to generate a "buffer zone" between the 
coarsening and refinement sweeps. It must be pointed out that no buffer zone is introduced explicitly but is implemented dynamically. The basic idea of the skip algorithm is to skip partial nodes at each coarsening sweep. The criterion was shown in Figure 3. Given an index of Voronoi cells, loop each vertex of the polygonal cell, a pair of neighbor cells cannot be processed continuously twice, need to at least skip once. For example, current iteration is working on cell $j$, loop the vertex $i$, the red region stands for its Delaunay triangle with $j, j+1$, and $j+2$ being three neighbor Voronoi cells. Now loop the next vertex which is the $(i+1)$ node, the corresponding Delaunay triangle comprises of cell $j, j+2$, and $k+1$. However, $j$ and $j+2$ two neighbor cells have participated in vertex $i$, it cannot be involved continuously. If continue to loop the next vertex which is the $(i+2)$ node, the corresponding three cells will be $k, k+1$, and $j$, so it works (only the cell $j$ continuously involved). The above operation will continue in the domain until the generation of a buffer zone.

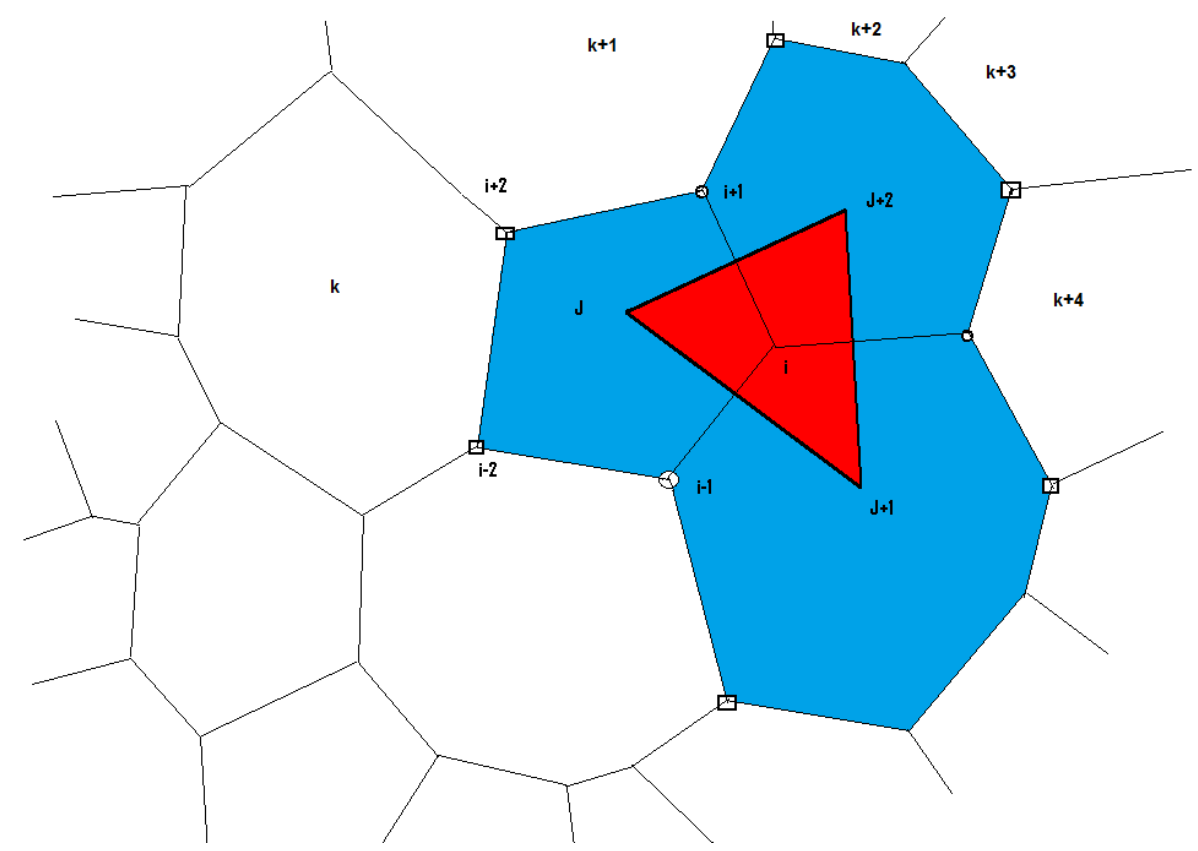

Figure 3: The criterion used for the implementation of the skip algorithm to generate a buffer zone for coarsening and refinement sweeps. 
The implementation of the coarsening algorithm is simply through a first-encounter-firstprocess rule. For example, in the marking subset of generators (Fig. 4b), we counter-clock wisely check each generator, and use the skip algorithm to go through the vertices of the Voronoi cell (i.e. the generator). If a vertex is marked to be coarsened, all vertices that directly connect this vertex will be determined to be skipped or not at current sweep. It leads to a list of small red squares in Fig. 4c, from which it is seen that each small red square is shared by its neighbor generators. After using the coarsening sweep, it will lead to a list of new generators as shown by the " $X$ 's" in Fig. 4d. All those small blue squares will not join the current coarsening sweep. 


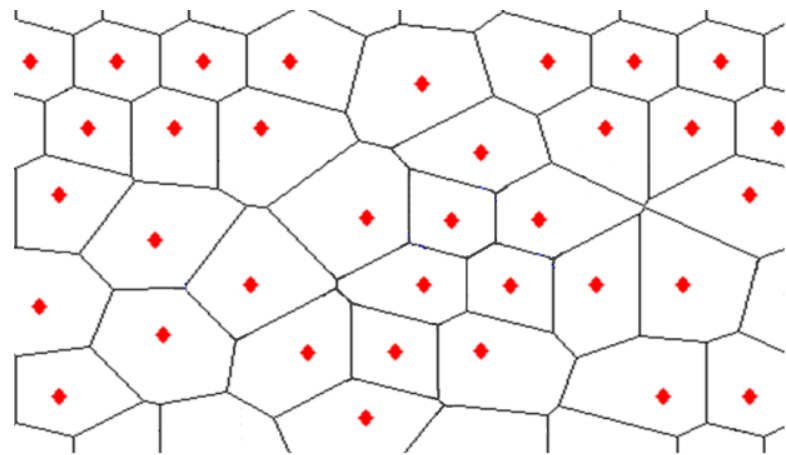

(a)

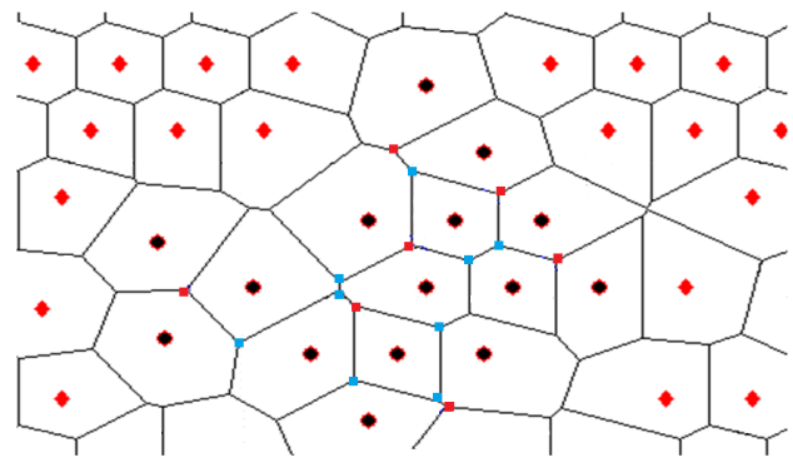

(c)

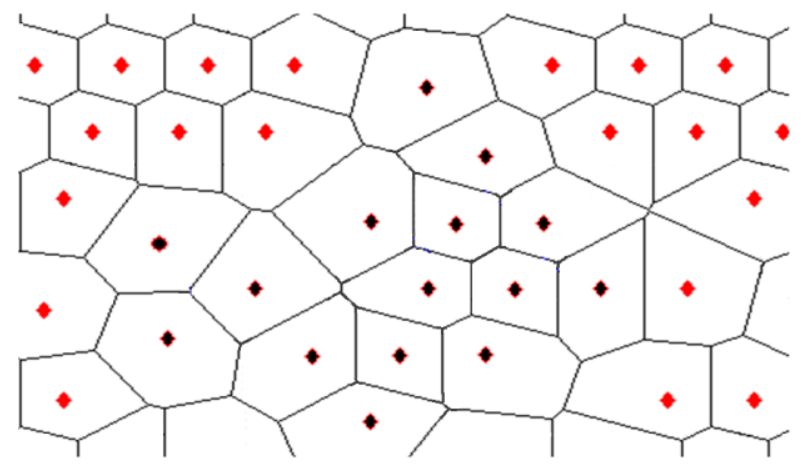

(b)

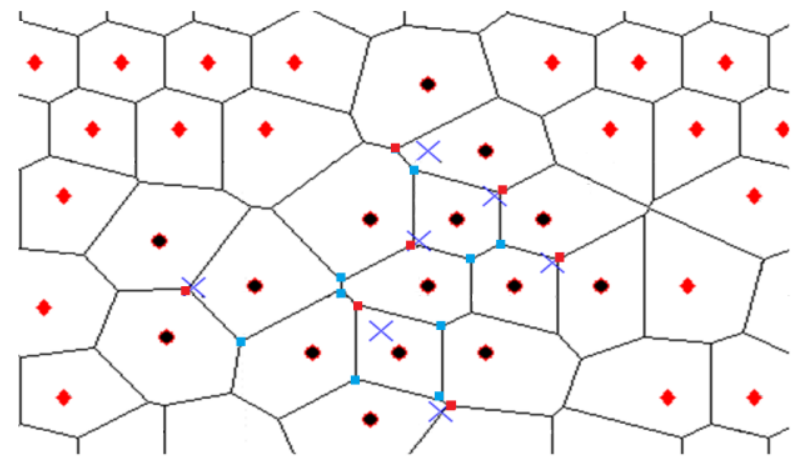

(d)

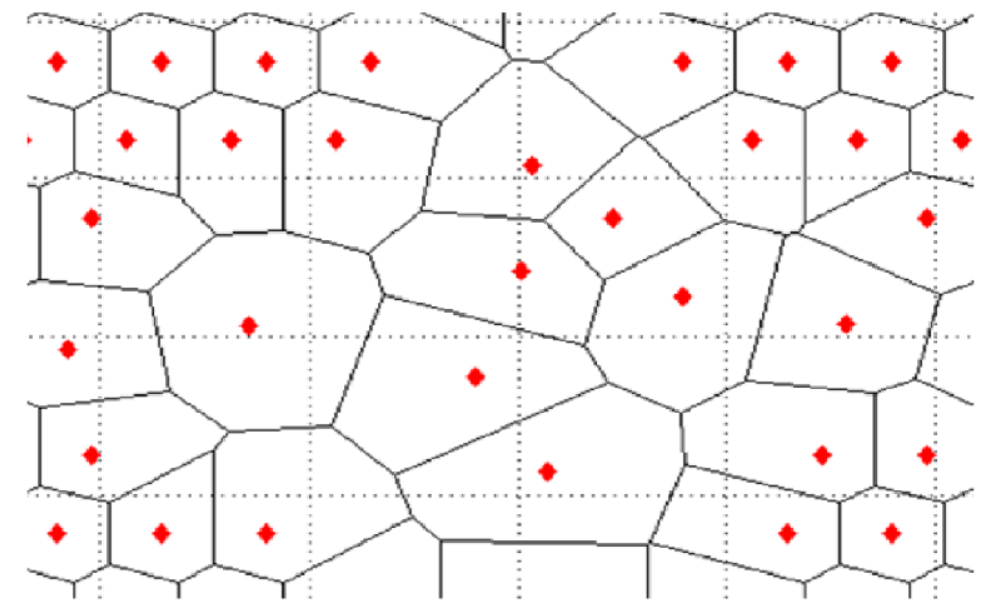

(e)

Figure 4: The skip algorithm for the coarsening sweeps of the polygonal mesh: (a) initial mesh, (b) marking subset, (c) determination of skipped generators, (d) final step of the algorithm, and (e) the new mesh. Filled black rhombuses are the coarsening region, blue " $X$ 's" denote new generators to be added later on during the mesh generation step, filled red squares are the vertices that have been processed for coarsening while the filled blue squares have been skipped in order to effectively generate a buffer zone, and the red rhombuses are the Voronoi regions that do not participate the process in current coarsening sweep. 
Refinement process. After the coarsening sweeps are done, the refinement will be invoked in two ways: synchronous and asynchronous processes. If the coarsening and refinement sweeps are conducted on the same mesh $M^{n}$, we call it synchronous process, otherwise an asynchronous process in which coarsening on current mesh $M^{n}$ following by several global mesh regulation operations, then the refinement on the new mesh $M^{n+1}$. Numerical experiments has demonstrated very tiny difference between two invoking operations in terms of volume equidistributions. The only difference is asynchronous process leads to smooth transition of meshes. Following a similar way as the coarsening process, we define the upper bound of mesh resolution $\bar{M}=\varphi_{r} \frac{1}{R} \int_{\Omega} \sqrt{f(x, y)} d A$

where the threshold parameter $\varphi_{r}$ is chosen either a priori or obtained a posterior from numerical solution of PDE. Without loss of generality, a constant such as 1.125 is used in numerical experiments below. We loop every vertex in the domain $\Omega$, calculate the real mesh resolution, and construct subset of generators such that

$\frac{\sqrt{f\left(x_{1}, y_{1}\right)}+\sqrt{f\left(x_{2}, y_{2}\right)}+\sqrt{f\left(x_{3}, y_{3}\right)}}{3} A_{123}>\varphi_{r} \frac{1}{R} \int_{\Omega} \sqrt{f(x, y)} d A$

The generators in the subset will be marked for refinement process. New generators will be inserted into the marked region. Figure 5 illustrates a typical refinement sweep. 


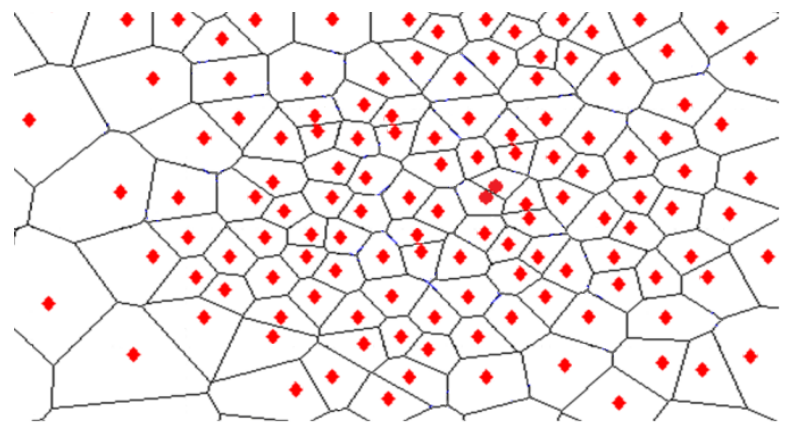

(a)

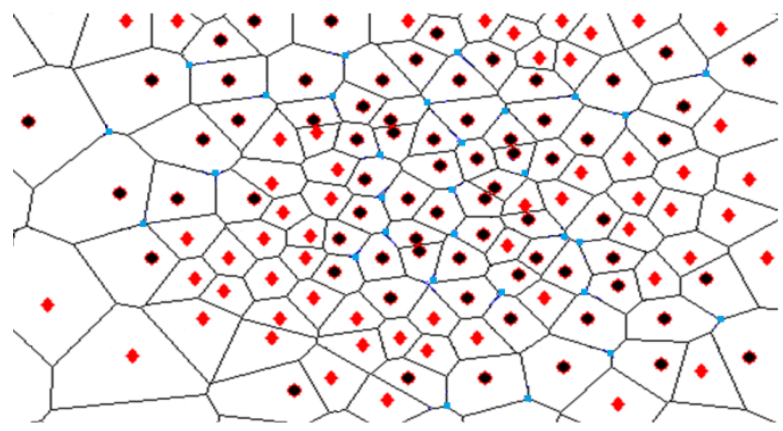

(c)

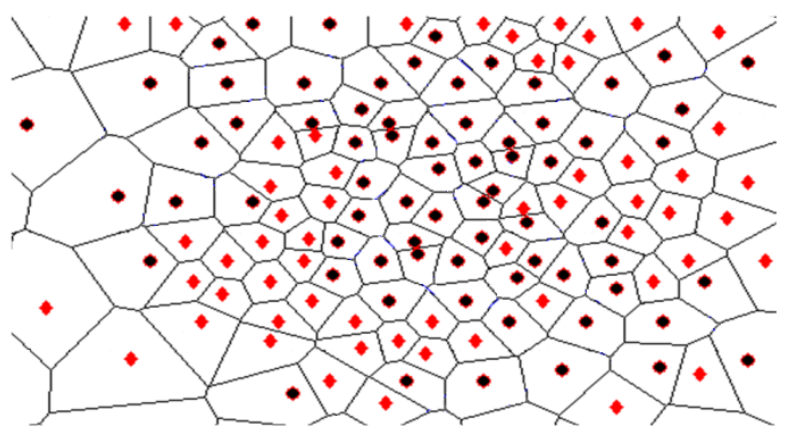

(b)

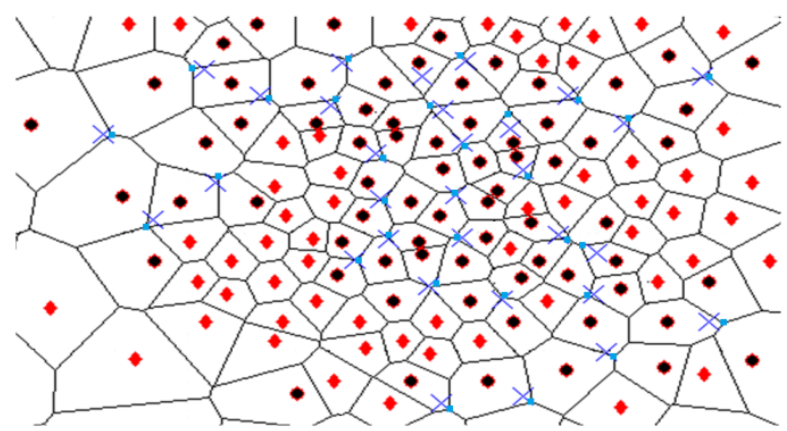

(d)

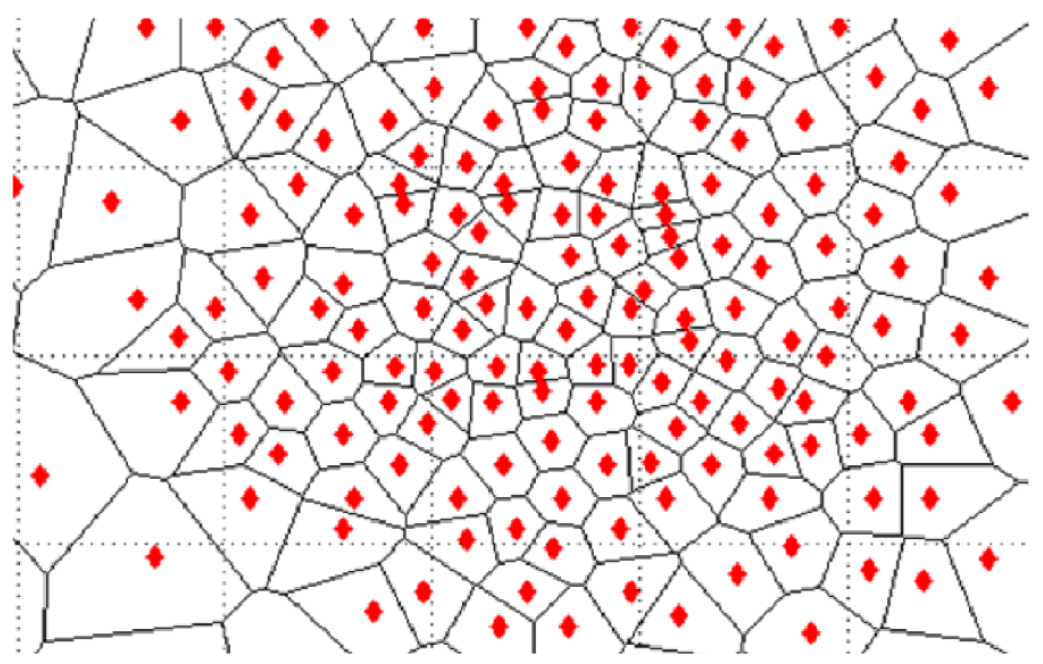

(e)

Figure 5: Implementation of a refinement sweep: (a) an initial mesh, (b) the marked region for refinement, (c) the small blue squares show the vertices to be refined, (d) the locations of added new generators are illustrated by the blue " $x$ 's", and (e) the new mesh.

Boundary nodes. If the given bandwidth between the lower bound and the upper bound is large enough the adaptive process will effectively converge to correct mesh resolution within mostly 10 sweep iterations (coarsening plus refinement) for the examples shown in this paper. The above marking algorithm for coarsening and refinement sweeps is default for internal 
Voronoi cells. The process for boundary region is similar by introducing the so-called "approximate Delaunay triangle" idea. The following example outlines the design of the algorithm (Figure 6). Basically there are two types of boundary nodes (i.e. vertices) in 2D. The first one is a node shared by three neighbor Voronoi cells and the boundary node can be handled in the same way as internal nodes (Figure 6a). The second type is a boundary node shared only by two neighbor Voronoi cells which needs special operation (Figure 6b). In Figure 6a, the shadow region is a real Delaunay triangle, "1", "2" and "3" are three Voronoi generators; however, in Figure 6b, the shadow triangle unnecessarily satisfies the definition and geometrical features of Delaunay triangulation. It is called as "approximate Delaunay triangle" with the boundary node to be one generator. The mesh resolution metric is the same as before by simply replacing the coordinate $\left(x_{2}, y_{2}\right)$ in Eqns. (2), (4) and (7) with the boundary node coordinate $\left(x_{2}^{*}, y_{2}^{*}\right)$, respectively for the marking strategy used in the coarsening and refinement here. It is seen that the proposed boundary adaptation is simple and easy to implement compared to complicated algorithms such as projecting or lifting methods available in literature about Voronoi tessellations (e.g., Ju et al. 2006).

(a)

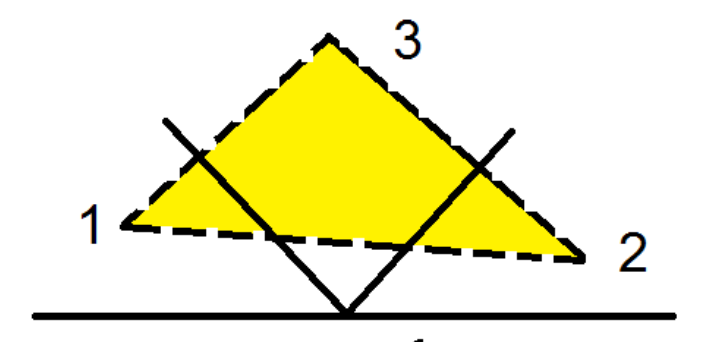

(b)

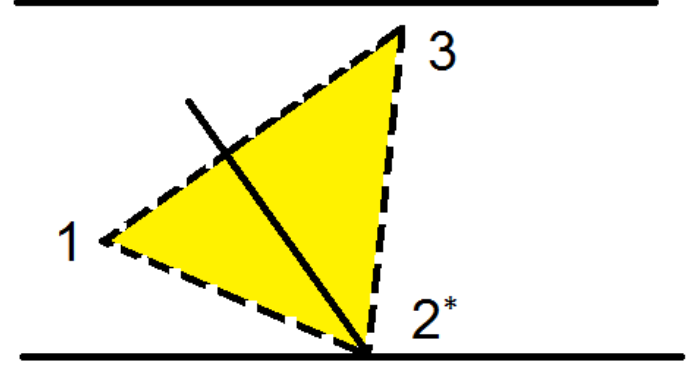


Figure 6: Delaunay triangles and the boundary nodes: (a) the boundary node is treated in the same way as internal nodes; (b) an approximate Delaunay triangle method is used for the marking strategy to handle the boundary node " 2 "”.

\subsection{A new moving strategy (local smoothing)}

Numerical experiments have shown that the convergence of the above $h$-adaptive process work well for most scenarios except very few cases in which the bandwidth of the goal mesh resolution $[\underline{M}, \bar{M}]$ is very narrow. The adaptation iteration could become drastically slow or even not convergent. A simple case is used to explain the challenge. For instance, after several iterations, a mesh with 20 generators in the domain is obtained (they are almost equi-distributed), however, it is still too dense as for the threshold parameter $\varphi_{c}$ which allow the coarsening sweep to be invoked. After the coarsening process (e.g. a single coarsening iteration), there are approximately 10 generators remaining in the domain, but now it is too coarse as for the threshold parameter $\varphi_{r}$. The dilemma is that neither pure $h$-adaptive nor pure $r$-adaptive can solve this problem alone. In order to fix this problem, a novel moving strategy (hereafter referred to as the local smoothing) with a flavor of double thresholds for the marking strategy is described below.

The local moving strategy is illustrated in Figure 7. Supposed that Node $i$ does not satisfy the goal mesh resolution, the three neighbor Voronoi cells denoted by $j, j+1, j+2$, respectively (the red triangle) will be marked. The following formula is then applied to determine the new locations of the three generators associated to the Voronoi cells

$x_{j}^{\prime}=x_{n i}+\frac{1}{\text { ratio }} \times\left(x_{j}-x_{n i}\right)$

$y_{j}^{\prime}=y_{n i}+\frac{1}{\text { ratio }} \times\left(y_{j}-y_{n i}\right)$

where $\left(x_{n i}, y_{n i}\right)$ is the coordinate of Node $i$ in Figure 7. The aspect ratio is defined as follows 
ratio $= \begin{cases}M / \bar{M} & \text { above the bandwidth } \\ M / \underline{M} & \text { below the bandwidth }\end{cases}$

The adjusted generators are referred to as $j^{\prime}, j+1^{\prime}$, and $j+2^{\prime}$, respectively (the blue triangle).

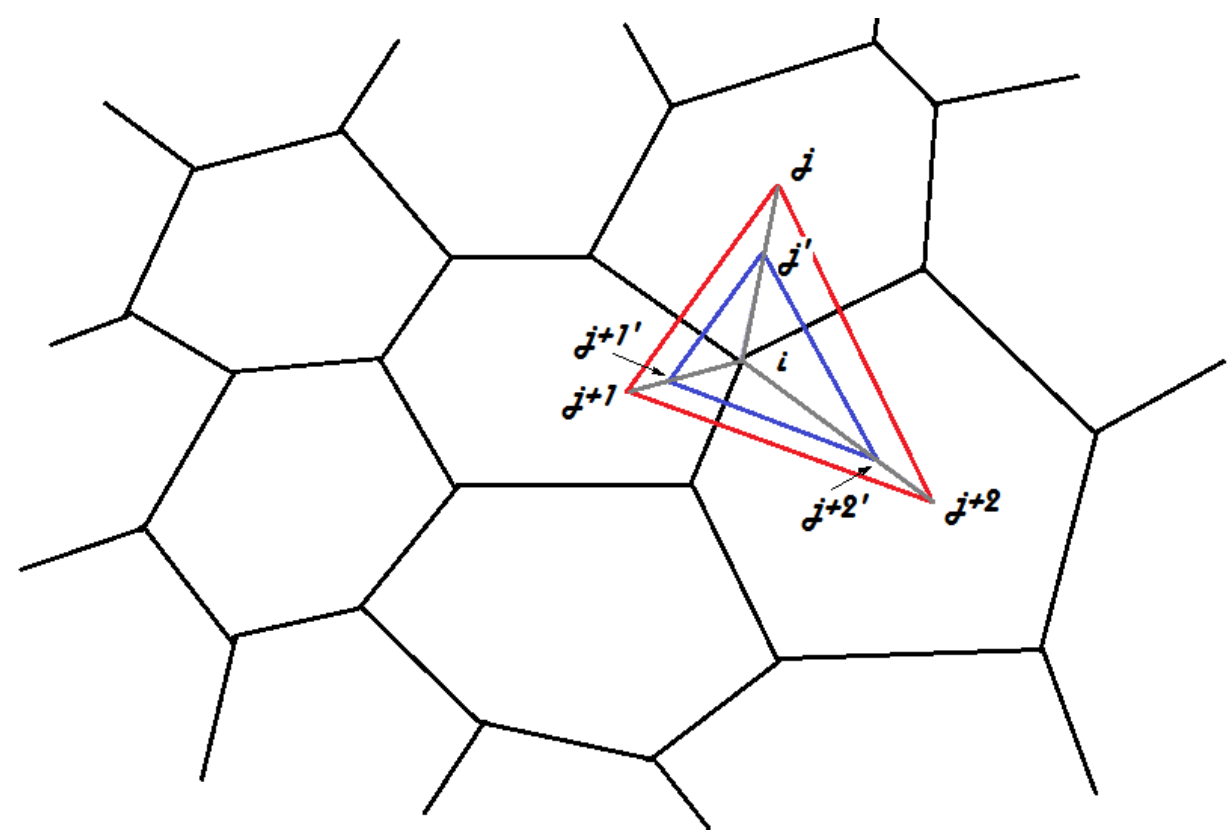

Figure 7: Schematic of the new local moving strategy associated with the double threshold marking algorithm. The original Delaunay triangle is illustrated by the red color, while the adjusted triangle by the blue color.

The following double threshold marking algorithm is used to determine the subset of nodes (i.e. vertices of Voronoi cells) for invoking of the local smoothing process. The underlying idea is to isolate the subset of nodes that could jump out of the bandwidth of the goal mesh resolution for a single adaptive sweep (either the coarsening or the refinement), then call the local smoothing process. Figure 8 illustrates the double threshold marking algorithm in 2D. 


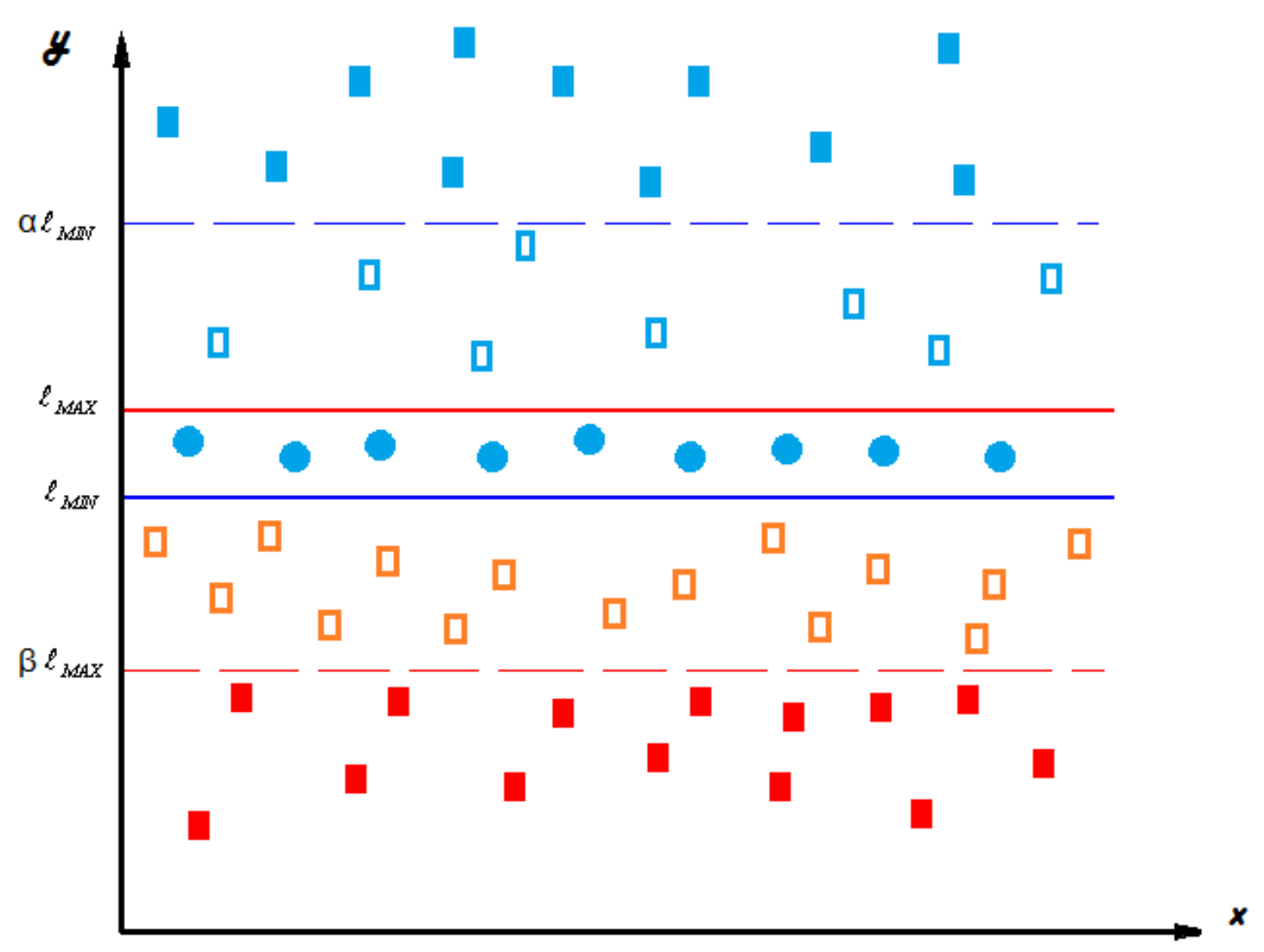

Figure 8: Schematic of the double threshold marking algorithm for local smoothing process. The horizontal axis denotes the index of each node in the entire region, while the vertical axis denotes the real mesh resolution of each node.

The two solid lines are the original bounds, $\ell_{M A X}=\bar{M}, \ell_{M I N}=\underline{M}$, while two dash lines stand for the extended bounds. Two control factors $\alpha(\geq 1)$ and $\beta(\leq 1)$ are introduced here to accurately determined the margin of the extended bandwidth. Here is how to compute the two factors, $\alpha$ is the minimum value of the rate of change of real mesh resolution for single refinement iteration in the domain, $\beta$ is the maximum value of the rate of change of real mesh resolution for single coarsening iteration in the domain. For instance, there is a subset $\left\{v_{i} \mid M_{v_{i}}<\right.$ $\left.\ell_{M I N}, i=1, \cdots, n_{r}\right\}$ for refinement process, $n_{r}$ is the size of the subset. $\alpha=\min \left\{M_{v_{i}}^{\text {new }} /\right.$ $\left.M_{v_{i}}^{\text {old }}\right\}, i=1, \cdots, n_{r}$. On the contrary, $\beta=\max \left\{M_{v_{i}}^{\text {new }} / M_{v_{i}}^{\text {old }}\right\}, i=1, \cdots, n_{c}$, for the subset $\left\{v_{i} \mid M_{v_{i}}>\ell_{M A X}, i=1, \cdots, n_{c}\right\}$ for coarsening process, $n_{c}$ is the size of the subset. In such a 
way, all nodes denoted by solid squares will be moved into the extended bandwidth $\left[\beta \ell_{M A X}, \alpha \ell_{M I N}\right]$. If a node is already in the inner bandwidth (the smaller one), no optimization operation is needed (the solid circles in Figure 8). For other nodes fell into the gap between smaller bandwidth and the extended bandwidth (the open squares in Figure 8) will go through the local smoothing iteration subsequently. In such a way, the final mesh resolution will reach to the goal mesh resolution. It is important to point out that the two factors $\alpha$ and $\beta$ are automatically determined rather than two ad-hoc constants used in the numerical experiments for the sake of simplicity.

\subsection{The weighted smoothing algorithm (global mesh regulation)}

The weighted smoothing algorithm is based on centroidal Voronoi tessellation (CVT) technique. Compared to the complicated data structure used in the $h$-adaptive algorithm, the weighted smoothing data structure is relatively simple. For the sake of completeness, the smoothing (i.e., $r$-adaptive) method is summarized below.

Given a density function (e.g., distribution of a geometrical or physical parameter) defined in the domain $\Omega$, for any Voronoi region, the centroid $z^{*}$ is computed by

$\bar{x}=\frac{\iint \rho(x, y) x d A}{\iint \rho(x, y) d A}, \quad \bar{y}=\frac{\iint \rho(x, y) y d A}{\iint \rho(x, y) d A}$

A Voronoi tessellation is referred to as a centroidal Voronoi tessellation (CVT) if and only if the points $\left\{z_{i}\right\}_{i=1}^{n} \in \Omega$ which serve as the generators of the associated Voronoi cells are also the centroids of these cells

$z_{i}=z_{i}^{*}, \quad i=1, \cdots, n$ 
It is desirable to let the centroids (i.e., the center-of-mass for constant density function) lies close to the mesh generator Voronoi tessellations, because this will lead to the minimum of energy distribution (Du et al. 1999; Du and Gunzburger 2002). In order to obtain CVTs, the so-called “Lloyd's iteration" has been widely used for generation of CVTs (Lloyd 1982). In the hybrid $h$ adaptive algorithm, the CVTs combined with Lloyd's iteration is serving as the weighted smoothing process (hereafter referred to as the global mesh regulation). In each sweep of the weighted smoothing, the centroids of Voronoi cells are obtained which are used as the new generators to generate new mesh, a sequence of optimization of Voronoi cells. The cornerstone for the implementation of the weighted smoothing algorithm is the calculation of the weighted centers of Voronoi cells which will be described in detail in Part II: Application, a separate paper, for finite volume methodology.

\subsection{Implementation of the complete algorithm}

A complete procedure for the hybrid $h$-adaptive algorithm is "coarsening $\rightarrow$ local smoothing and/or global mesh regulation $\rightarrow$ refinment $\rightarrow$ global mesh regulation." The number of iteration depends on the goal mesh resolution and the bandwidth requirement of the optimization. Especially, if the upper bound and the lower bound of the bandwidth are close to each other, the iteration will converge asymptotically to a straight line in 2D domain. The implementation of the iteration process can be outlined below

1. Given the Voronoi mesh $V^{n}$ at the sweep level $n$, calculate the real mesh resolution $\overline{M_{k}}, k=$ $1, \cdots, n_{v}$ at every node in the domain, where $n_{v}$ stands for the total number of the nodes of the mesh. The boundary node algorithm is applied for those vertices on the boundaries. Record the resolution information with every node and the connection of Voronoi cell that the node belongs to. 
2. Loop every node in the domain, compare its real mesh resolution $\widetilde{M}_{k}$ with the lower bound of goal mesh resolution $\underline{M}$, if it is less than the lower bound, call the marking algorithm for coarsening as well as the skip algorithm. If not, compare the real mesh resolution to the upper bound $\bar{M}$. Call the marking algorithm for refinement process as well as the skip algorithm if the real mesh resolution is out of bound of $\bar{M}$. Otherwise go to Step3.

3. Compare the real mesh resolution with the two sub-bandwidths to determine control factors $\alpha, \beta$. If mesh resolution falls into the gaps between outer bandwidth and inner bandwidth, invoke the local smoothing algorithm by calling the double threshold marking algorithm.

4. Call global mesh regulation process if asynchronous process is required (the weighted smoothing or CVT $r$-adaptive)

5. Generate the new list of generators and the arrays of corresponding coordinates of generators. Call Voronoi tessellation and its topological dual, the Delaunay triangulation algorithm to establish the new polygonal mesh $V^{n+1}$ at the sweep level $n+1$

6. Examine the equi-distribution requirement, if every node stratifies the goal mesh resolution, then stop and exit with the final goal mesh. Otherwise, go to Step 1 for the next sweep process.

\section{Metric Study and Numerical Test}

We chose four cases to test the robustness and effectiveness of the novel hybrid algorithm. The first case was designed to demonstrate the detailed evolution of mesh resolution, the volume equi-distribution error, and comparison during the adaptation iterations. The second case was undertaken to test the behavior of boundary node algorithm for both step function and the Noh's problem (1987). Time-independent test was employed in the third case while time-dependent 
tests were presented in the fourth case (both are heterogeneous problems) to demonstrate the capabilities of the hybrid adaptive algorithm. In order to quantify the quality of the generated meshes, the following volume equi-distribution error (in an averaged sense) was introduced

$$
\sqrt{\sum_{i=1}^{N_{n}}\left(\frac{g^{i} A^{i}}{M}-1\right)^{2}}
$$

where $N_{n}$ was the number of Delaunay triangles in the domain where $n$ denoted the number of generators (Eqn. 1), $M$ was the normalized volume equi-distribution defined in Eqn. (3), $g^{i} A^{i}$ is the discretized local volume equi-distribution given by the left hand side of Eqn. (2). Because the new mesh involved here was created by Voronoi tessellation technique, it automatically avoided mesh distortion problem as quantified by the aspect ratio, skewness, and specific global grid distortion metric (Delzanno et al. 2008; Chacon et al. 2011).

\subsection{Test the evolution of mesh resolution and the equi-distribution error}

Given an initial uniform or arbitrary distribution of generators in a unit square, the monitor function is provided by

$f(x, y)=|\sin (2 \pi x) \sin (2 \pi y)|+c_{0}$

which is non-negative symmetric in the domain with a very small positive number $c_{0}=10^{-6}$, two order of magnitude less than the lower bound of the mesh resolution (Figure 9). The control factors are chosen for demonstration only, for example, $\ell_{M A X}=\bar{M}=1.785 \times 10^{-4}, \alpha=\bar{M} / \underline{M}$, $\beta=1 / \alpha$, and $\ell_{M I N}=\underline{M}=5.625 \times 10^{-5}$, so $\alpha=3.1733, \beta=0.315$. In such a way, the double threshold marking strategy will regenerate and become the conventional single threshold marking approach. In default the numerical code will invoke the asynchronously adaptive process, for the purpose to demonstrate intermediate changes of the mesh clearly. In other words, 
the implementation for the adaptive sweeps will be initial uniform mesh $\rightarrow$ coarsening $\rightarrow$ global mesh regulation $\rightarrow$ refinement $\rightarrow$ global mesh regulation loop. The real mesh resolution evaluated at every node will be generated for equi-distribution error analysis. Figure 10 provides four snapshots of real mesh resolution, in which each point stands for a node. In each panel, the green line denotes the upper bound $\bar{M}$ and the blue line stands for the lower bound $M$. It is seen that the initial mesh does not satisfy the equi-distribution of mesh resolution given by the monitor metric. After the coarsening sweeps completed, there are no more nodes below the lower bound line (Fig. 10(b)). The real mesh resolution becomes more isotropic after the refinement sweeps (Fig. 10(c)). The final mesh is approximately optimal with respect to the monitor metric (Fig. 10(d)). Figure 11 illustrates corresponding four polygonal meshes obtained.

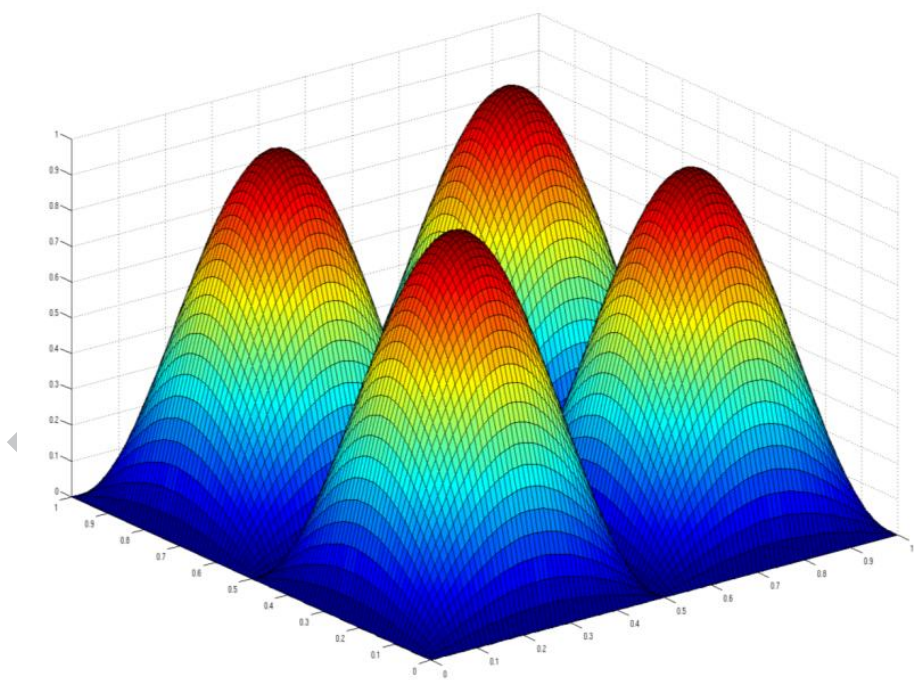

Figure 9: The monitor metric for the first test problem. Large value will lead to small area of polygonal cells (i.e., more generators will cluster) while small value will result in coarse mesh locally. 

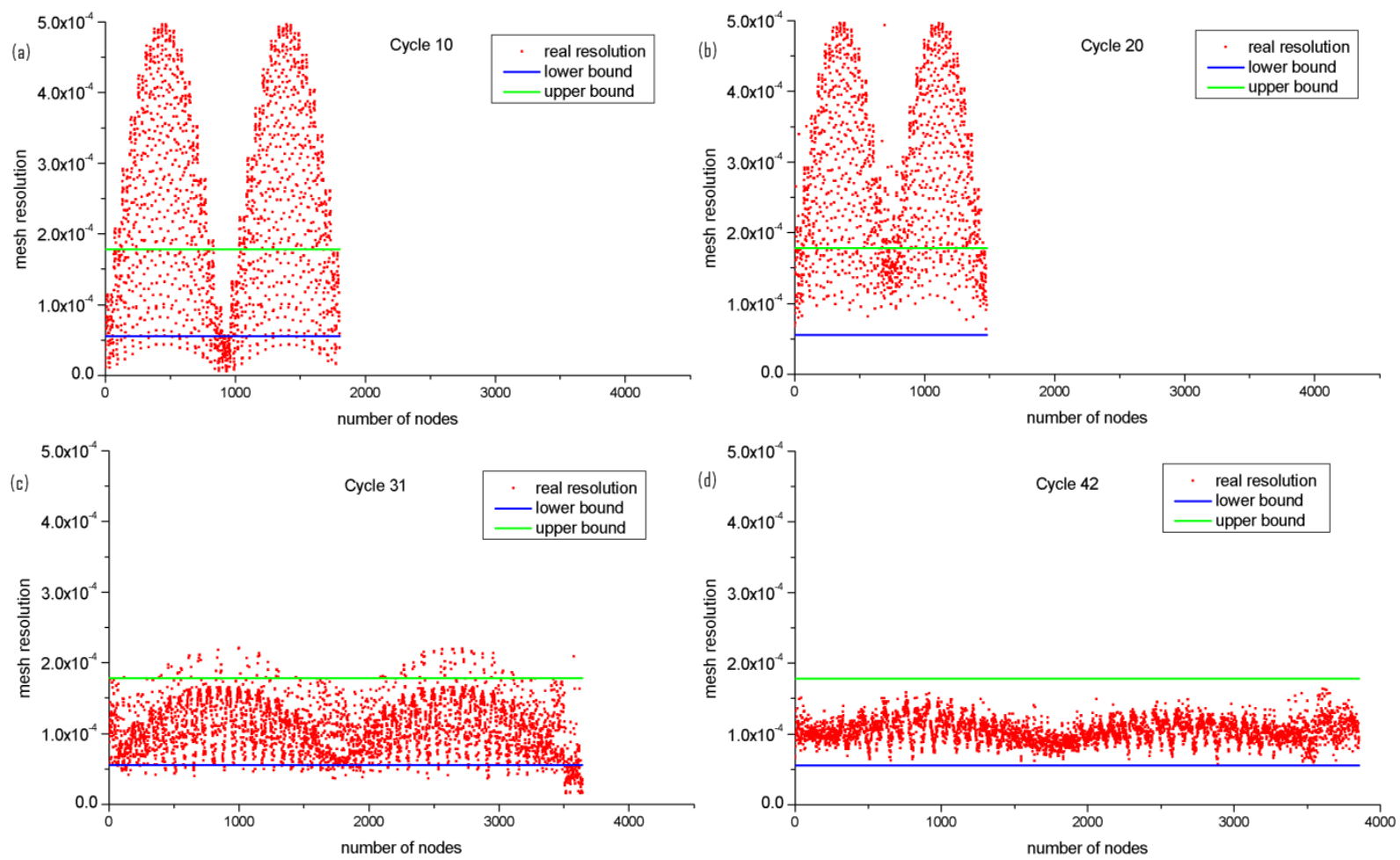

Figure 10: The evolution of real mesh resolution in the domain. (a) The initial uniform mesh; (b) the mesh distribution after coarsening sweeps were finished; (c) the mesh distribution after 3 refinement sweeps; and (d) the final mesh resolution after the global mesh regulation was completed. 

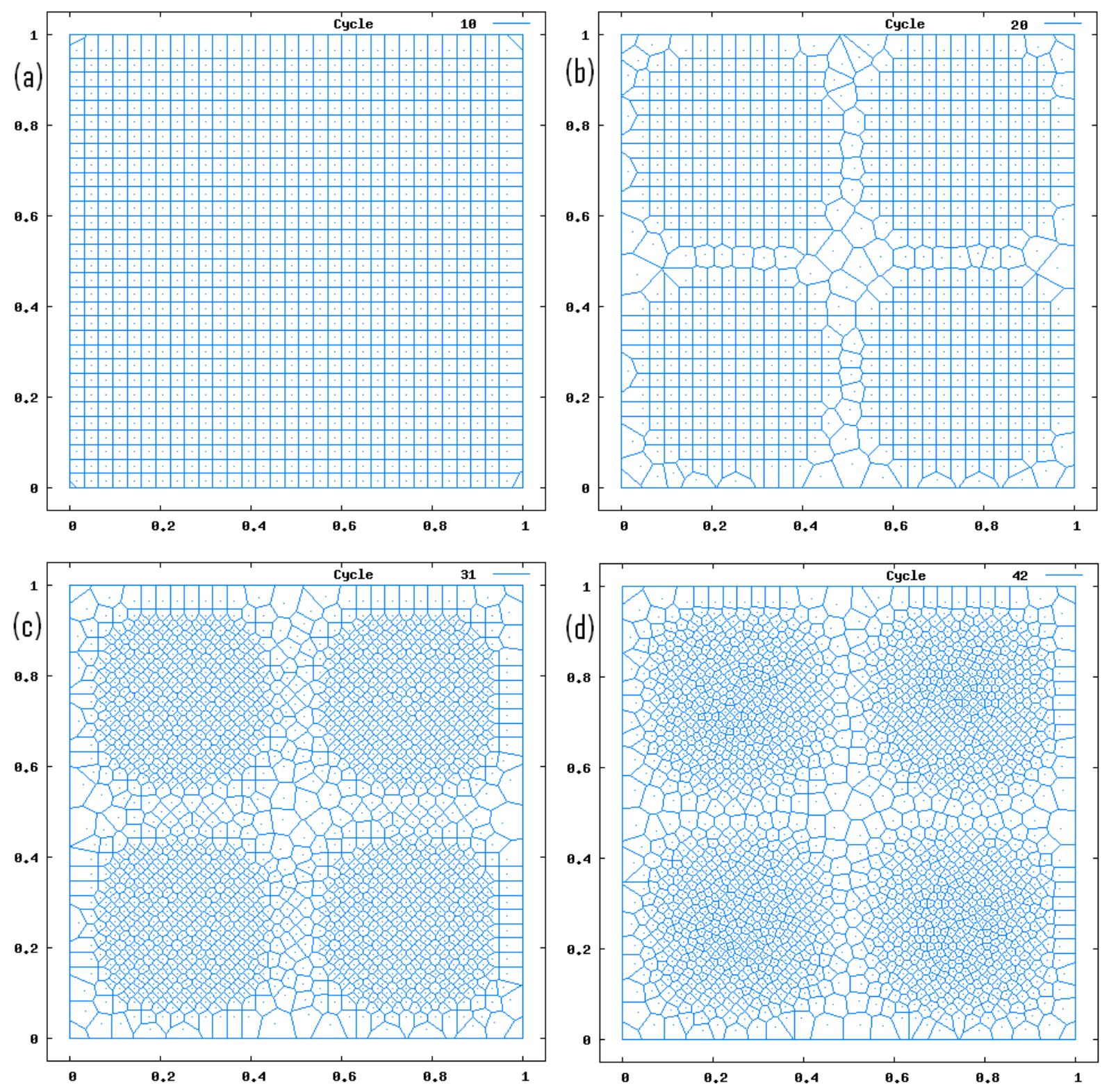

Figure 11: Four snapshots of the polygonal meshes obtained by the hybrid adaptation algorithm (here by asynchronous process). (a) The initial uniform mesh; (b) mesh obtained after coarsening; (c) optimized mesh after mesh regulation and 3 refinement sweeps; and (d) the final mesh that satisfies the equi-distribution requirement.

We now consider a smaller bandwidth of the goal mesh resolution by setting up $\bar{M}=$ $1.125 \times 10^{-4}, \underline{M}=8.75 \times 10^{-5}, \alpha=1.7$ and $\beta=0.5$, in such a way, the single threshold marking strategy was undertaken. Figure 12 shows the convergence of the adaptation iteration, and total 474 iterations have been conducted. The panel 12(d) illustrated the detailed distribution 
of the final mesh, a close-up of panel 12(c). To test the algorithm, we made a further shrink of the bandwidth of the goal mesh resolution by setting up $\bar{M}=5.625 \times 10^{-5}, \underline{M}=4.375 \times 10^{-5}$, $\alpha=1.7$, and $\beta=0.5$. This a much tougher case for which the final mesh converged after more than 700 iterations of adaptation (Figure 13). In this scenario the local smoothing will be invoked automatically. Given enough iterations of adaptation, the final mesh resolution can converge to a straight line in $2 \mathrm{D}$ asymptotically. This feature is particularly promising for simulating multi-scale problems with a variety of change of order of physical quantities without additional computational load.
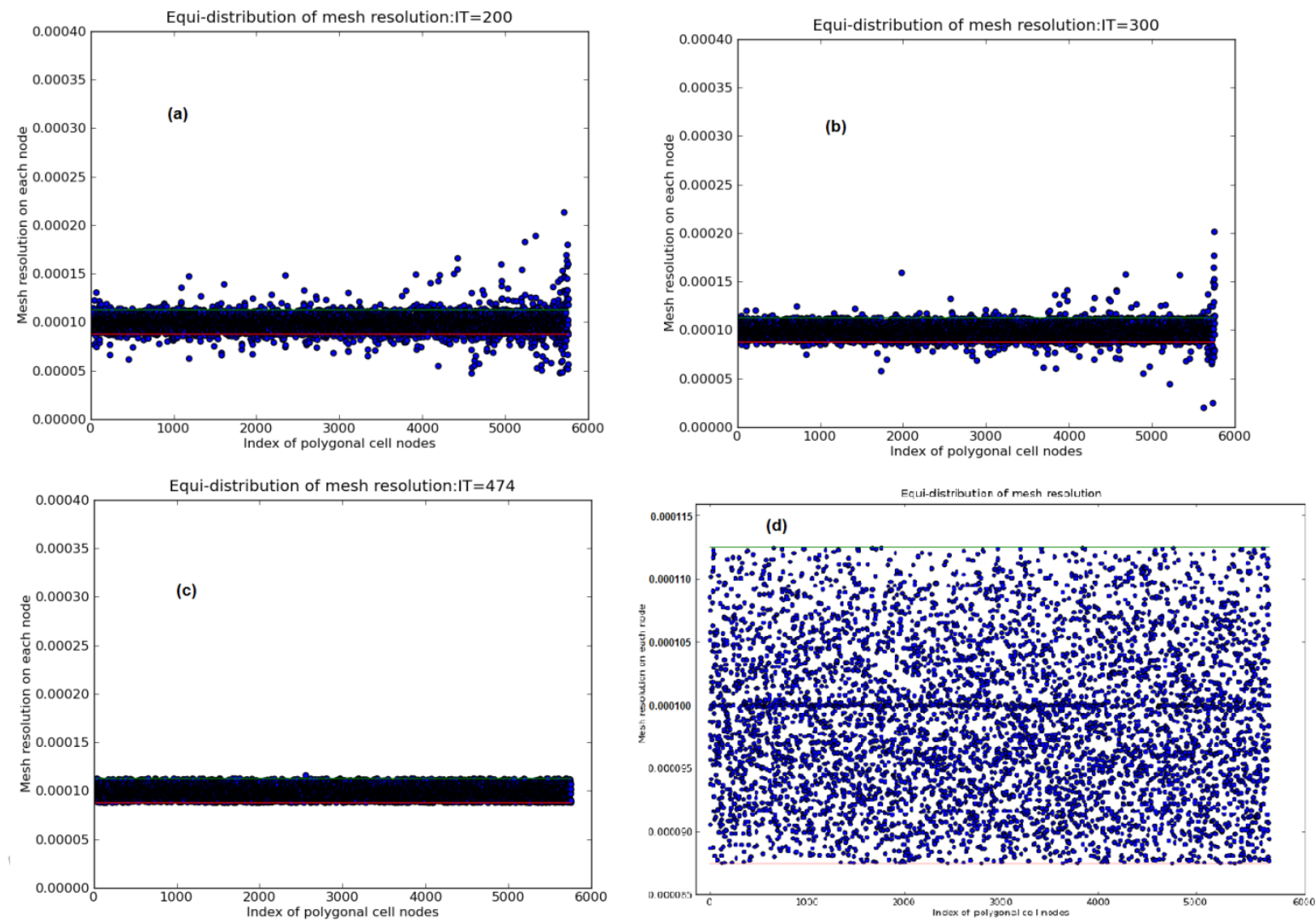

Figure 12: The final mesh resolution converged to arbitrary equi-distribution requirement after 474 iterations. The lower bound is $\underline{M}=8.75 \times 10^{-5}$ and the upper bound is $\bar{M}=1.125 \times 10^{-4}$. Panel (d) was a close-up view of panel (c), the final converged scenario. 


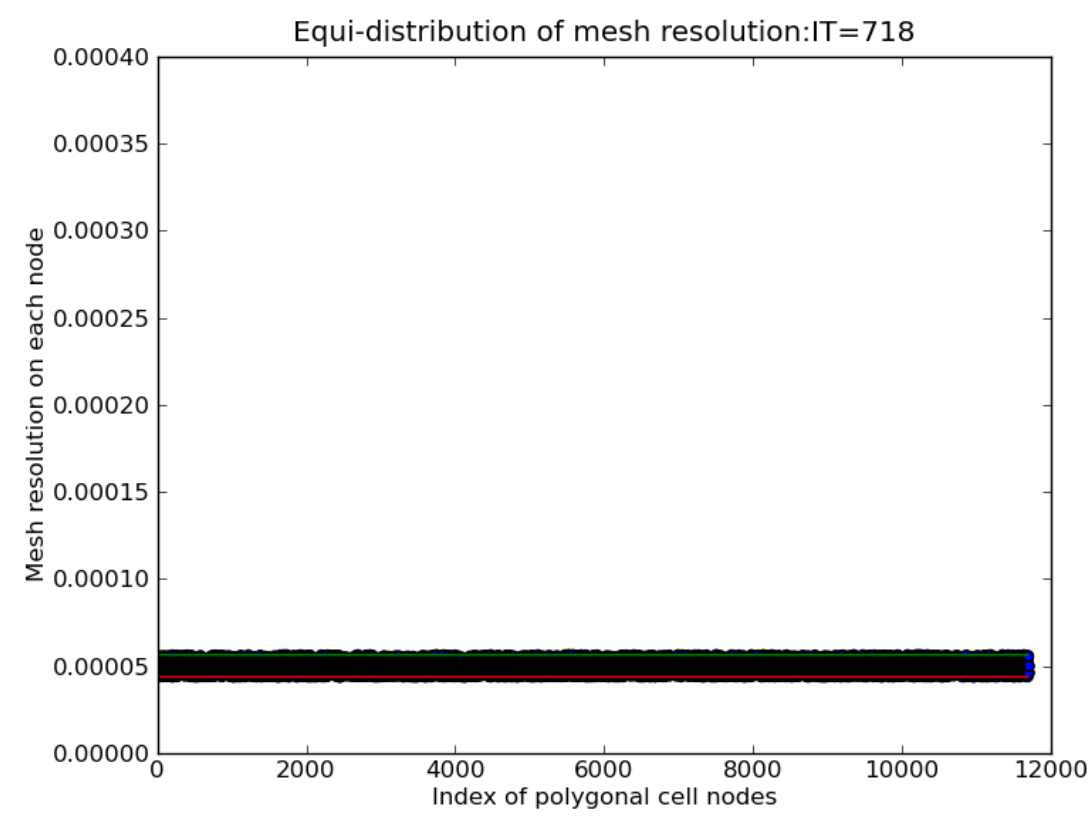

Figure 13: The finial mesh resolution converged to a much smaller resolution with other conditions the same as Figure 13. The lower bound was $\underline{M}=4.375 \times 10^{-5}$, and the upper bound was $\bar{M}=5.625 \times 10^{-5}$. The total number of iterations was 718 .

The above figures only demonstrated mesh resolution (i.e., the volume equi-distribution) during the adaptation iteration. It was not clear how well the volume equi-distribution error was in an averaged sense. Figure 14 and Figure 15 quantified the evolution of the equi-distribution errors with the hybrid iterations, one with the local smoothing algorithm (Fig. 14), the other without (Fig. 15). It is clearly seen the numerical error approach uniform distribution $\left(\mathcal{L}_{2}\right.$ norm) in Figure 14. However, the oscillation of the equi-distribution error $\left(\mathcal{L}_{2}\right.$ norm) shows a local mesh resolution problem due to Voronoi tessellations in Figure 15, if the local smoothing algorithm was not invoked during the adaptation. 


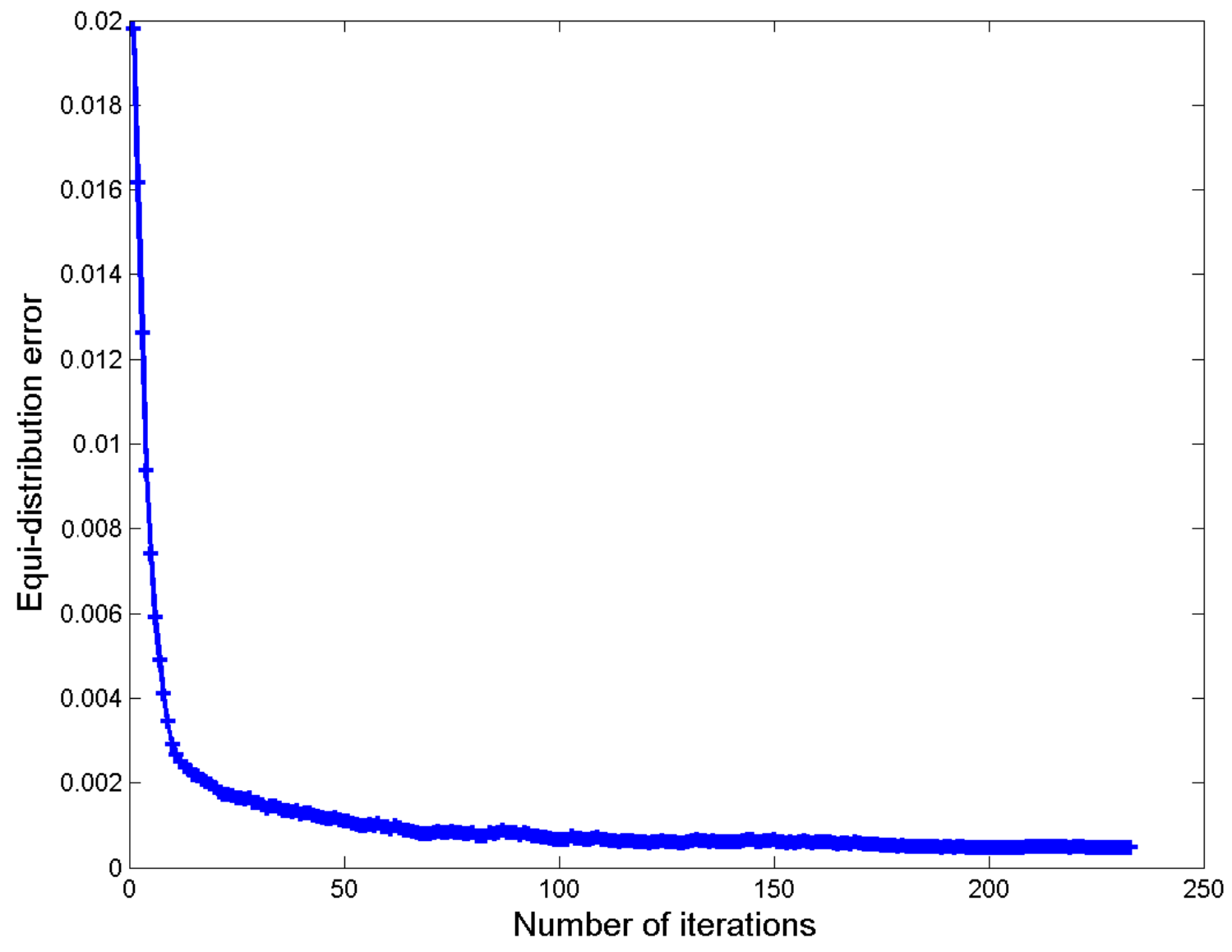

Figure 14: Evolution of the volume equi-distribution error in an averaged sense with the adaptation iterations associated with the case in Figure 13. The local smoothing algorithm was involved in the process which led to a monotonic decrease of the numerical error. 


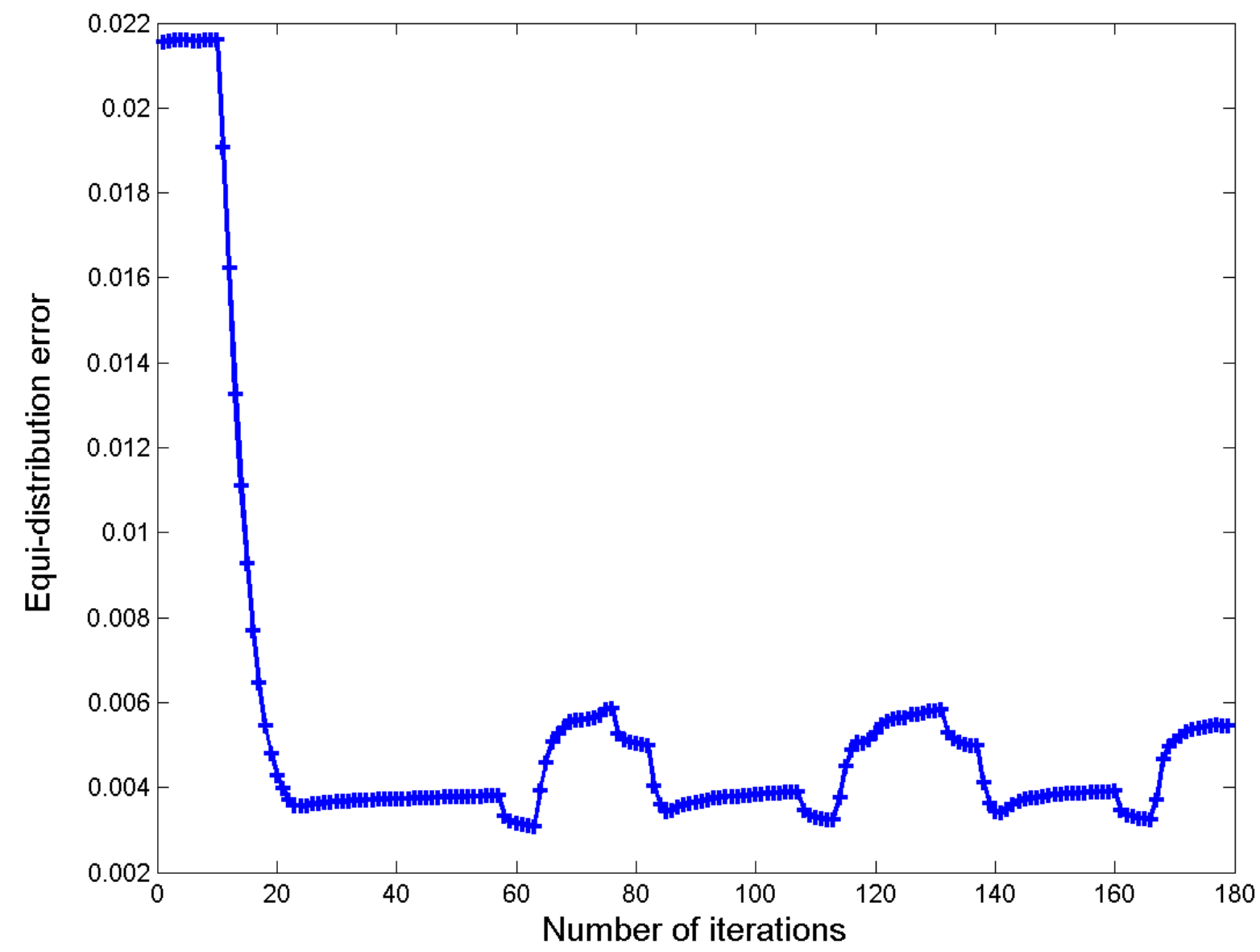

Figure 15: Oscillation of the volume equi-distribution error associated with the case as shown in Figure 13. The local smoothing algorithm was not turned on. The iteration did not converge in this test.

\subsection{Test the boundary node algorithm}

In above test, the region close to the boundary was coarsened with the implementation of the boundary node algorithm. Two examples here illustrate the difference with and without the boundary node algorithm and the capability for the mesh optimization in boundary regions. The following two scenarios are selected, one is for a step function and the other is for the Noh's problem (Noh 1987), a curved step function as shown by Eqns. (16) and (17), respectively,

$f(x, y)=\left\{\begin{array}{ccc}1 & 0.0 \leq x \leq 0.5, & 0.0 \leq y \leq 1.0 \\ 10 & 0.5 \leq x \leq 1.0, & 0.0 \leq y \leq 1.0\end{array}\right.$ 
$f(x, y)= \begin{cases}10 & 0.0 \leq \sqrt{x^{2}+y^{2}} \leq 0.5 \\ 1 & 0.5 \leq \sqrt{x^{2}+y^{2}} \leq 1.0\end{cases}$

Figure 16 demonstrates the difference before and after the applications of the algorithm for boundary nodes during the adaptive sweeps for the first example. Without the boundary node process there is significant distribution error in the region close to the boundary which did not satisfy the equi-distribution requirement of the monitor function. After imposing the iteration sweeps over the boundary nodes, the mesh distribution was consistent with the internal mesh distribution. Figure 17 presents a very well distribution of polygonal mesh for the Noh's problem. It is important to note that the number of generators lies on the boundary is fixed by using the boundary node algorithm. The reason for this is that it can avoid complicated process of adding (i.e., projection) or removing (i.e., lifting) generators on the boundaries, for example the lifting and projecting algorithm for constrained region adaptive procedure suggested by Ju et al. (2006). The justification for the simple boundary node algorithm is demonstrated clearly by the two examples. The volume equi-distribution error decreased monotonically with the number of iterations for the two examples (not shown here) which is similar to Figure 14. 

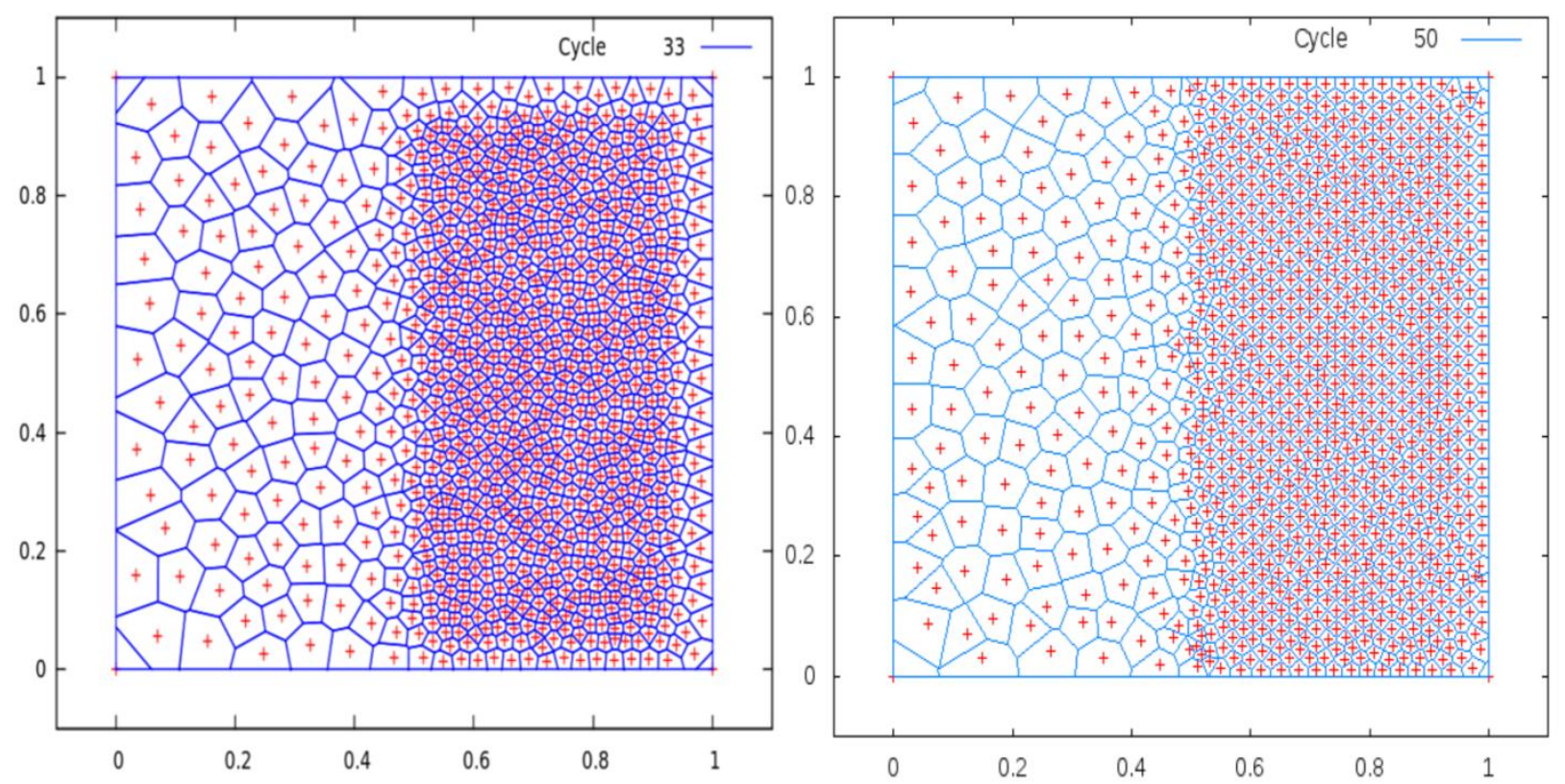

Figure 16: The $h$-adaptive sweeps of the domain to optimize the mesh without the boundary node algorithm (left panel) and with the boundary node algorithm (right panel). The bandwidth of mesh resolution was $1.25 \times 10^{-4}$. 


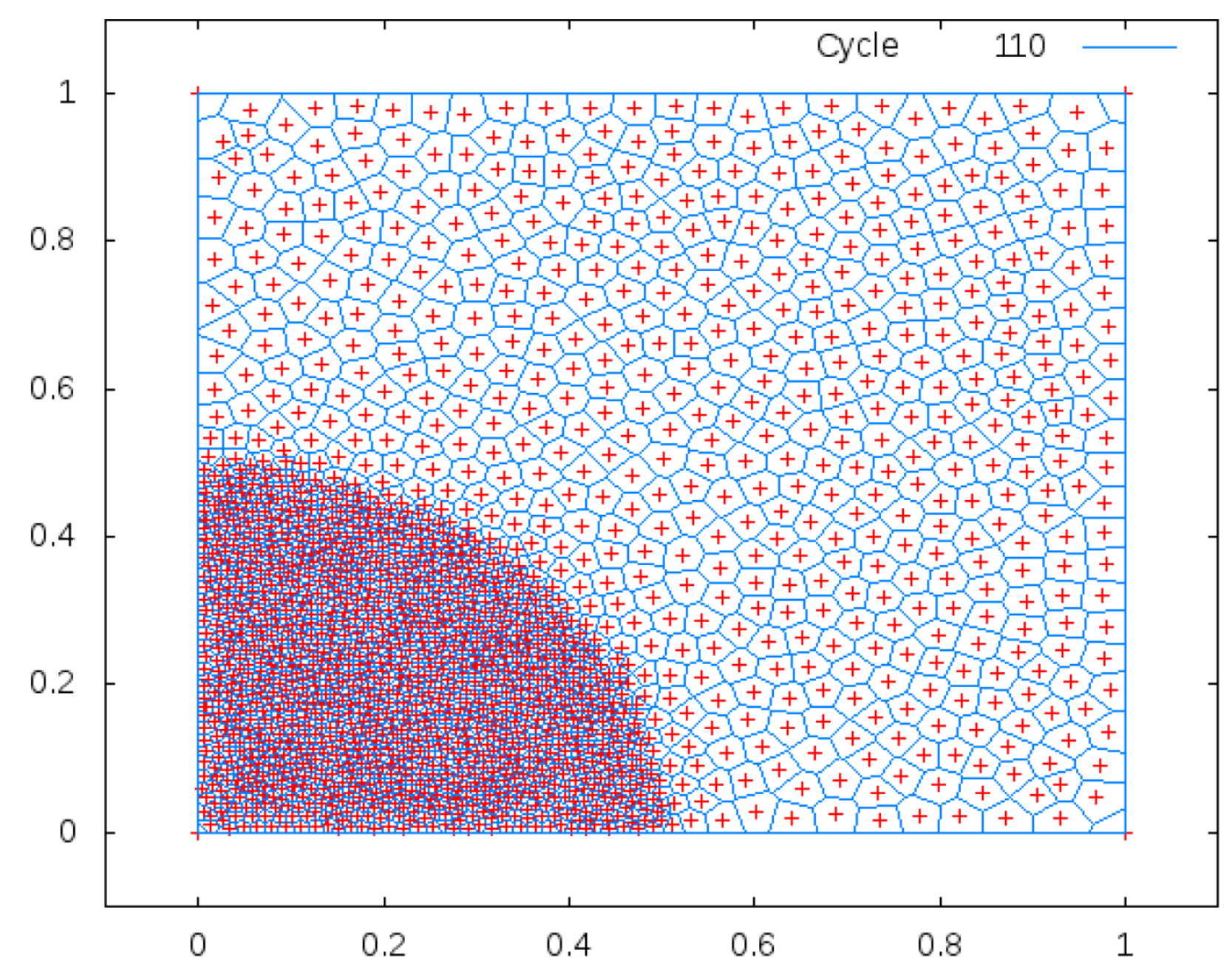

Figure 17: The $h$-adaptive sweeps over the domain for optimization of Noh's problem (Noh 1987) with the boundary node algorithm (initially there were 1000 generators in the domain). The bandwidth of mesh resolution was $1.25 \times 10^{-4}$.

\subsection{Test time-independent anisotropic distribution}

The following two examples stand for steady state problems which can be determined either a priori or from asymptotical solutions. The first example was taken from Huang (2005) for the anisotropic distribution of the field initially. Similar cases have also been used by other researchers (e.g., Vachal and Maire 2011). The monitor function in a unit square is given by

$f(x, y)=1.5-\tanh \left(100.0[y-0.5-0.25 \sin (2 \pi x)]^{2}\right)$ 
Figure 18 illustrates the monitor function for the goal mesh resolution, the higher value of mesh resolution, the denser generators are needed. Figure 19(a) shows an intermediate polygonal mesh after the coarsening sweeps as well as two the global mesh regulation sweeps in the domain. At this stage, no more generators would be removed from the domain. The final optimized mesh is shown on the right panel in Figure $\mathbf{1 9}$ after refinement sweeps as well as global mesh regulation. No local smoothing was needed here due to the relative large bandwidth. Again in this example, the asynchronous process has been undertaken for the coarsening and the refinement iterations. It is seen that a big advantage of the hybrid adaptive algorithm provides an effective alternative avenue compared to pure $r$-adaptive process conducted in Huang (2005), which avoided mesh distortion and mesh tangling problems by regeneration of new polygonal cells correspondingly. This feature is especially critical for modeling of complex flow problems for large gradient, transient, shock waves and deformation.

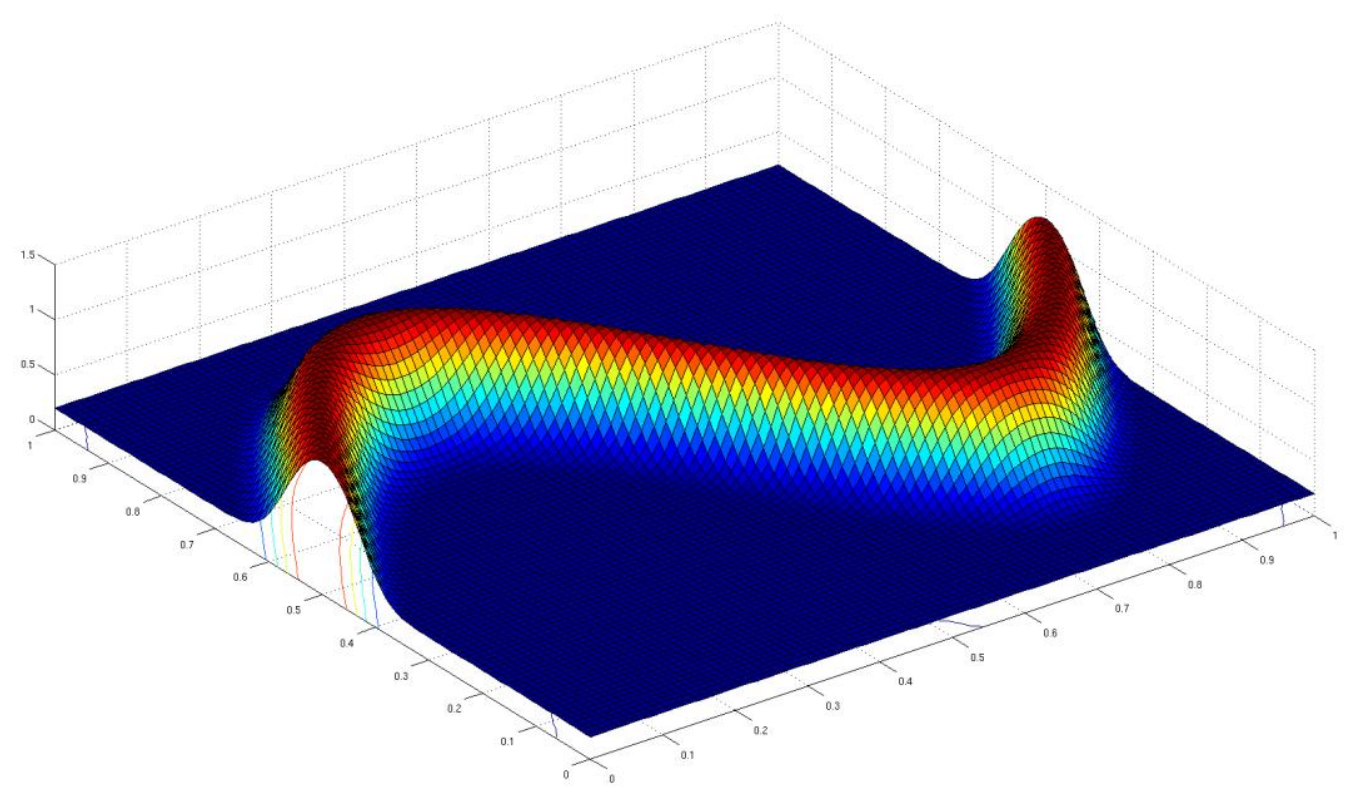

Figure 18: The monitor function in a unit square for the first test case adopted from Huang (2005). The red color region stands for dense mesh distribution needed. 

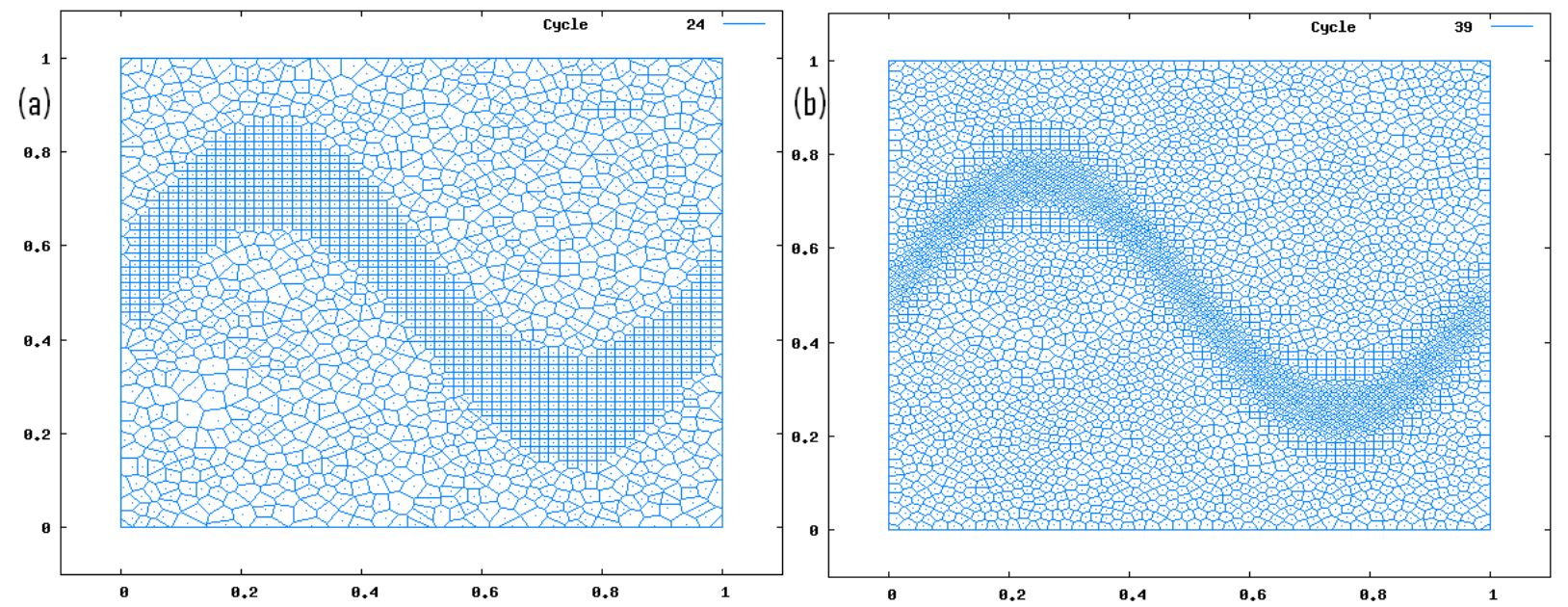

Figure 19: Two snapshots of the polygonal meshes: (a) an intermediate mesh after coarsening and two global mesh regulation sweeps (left panel); (b) the final mesh that met the goal mesh resolution (right panel). The bandwidth of mesh resolution was $5.0 \times 10^{-5}$.

The second example was adopted from Huang and Sun (2003). The monitor function is given by Eqn. (19) as shown in Figure 20 for the goal mesh resolution. Two snapshots of the polygonal meshes are demonstrated in Figure 21. The left panel was for an intermediate mesh after the coarsening and local smoothing finished while the right panel was the final polygonal mesh that met the equi-distribution requirement given by Eqn. (19). It is seen that a smooth transition of mesh size and clustering of generators in region was obtained. Figure 22 presents the evolution of volume equi-distribution error with the adaptation iteration for the two examples here. It is not surprising to see that it only took around 20 iterations (including global mesh regulation) to reach the goal mesh resolution. The equi-distribution error phenomenon at initial iterations was due to the initial number of generators and the monitor function. After 6-10 iterations, the averaged error rapidly decreased and converged.

$f(x, y)=2.0+\tanh \left(100.0\left[(x-0.5)^{2}+(y-0.5)^{2}-0.125\right]\right)$ 


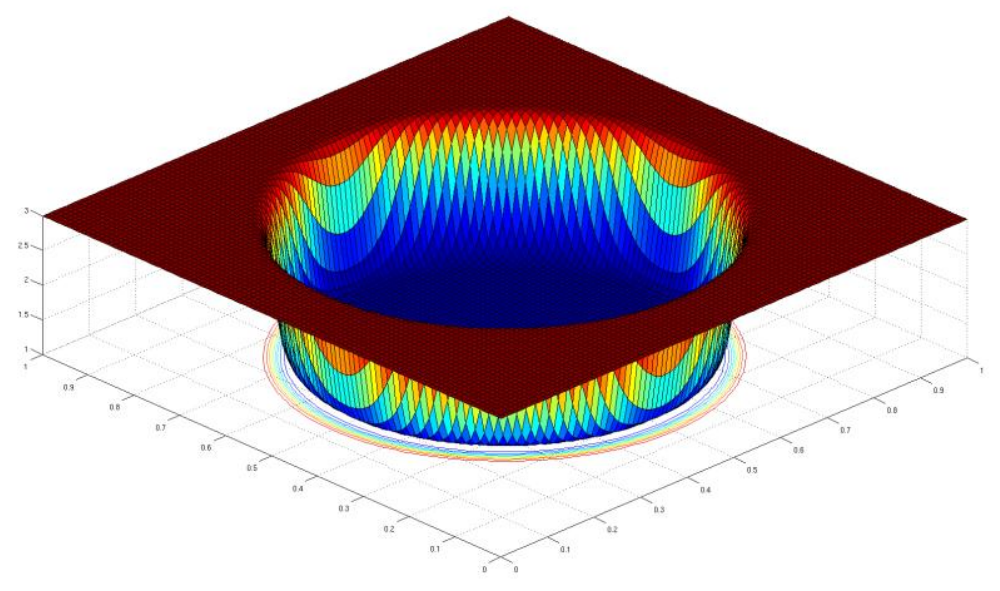

Figure 20: The goal mesh resolution for the second example adopted from Huang and Sun (2003). A big transient of mesh sizes is illustrated in the edge region of the circle.
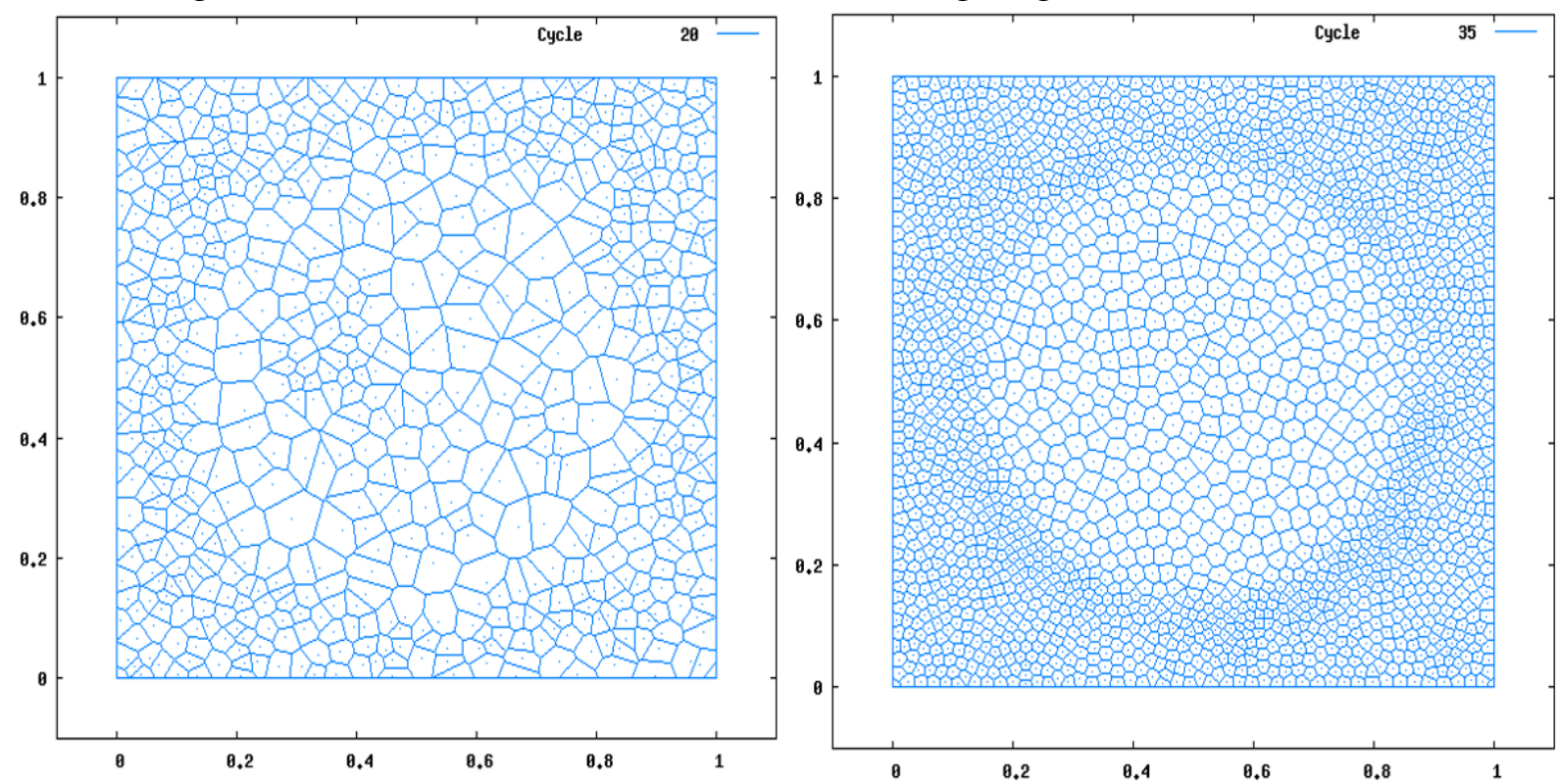

Figure 21: Two snapshots of the polygonal meshes obtained by the hybrid adaptive algorithm: an intermediate mesh after the coarsening and two global mesh regulation sweeps finished (left panel) and the final optimized polygonal mesh (right panel). The bandwidth of mesh resolution was $1.25 \times 10^{-4}$. 

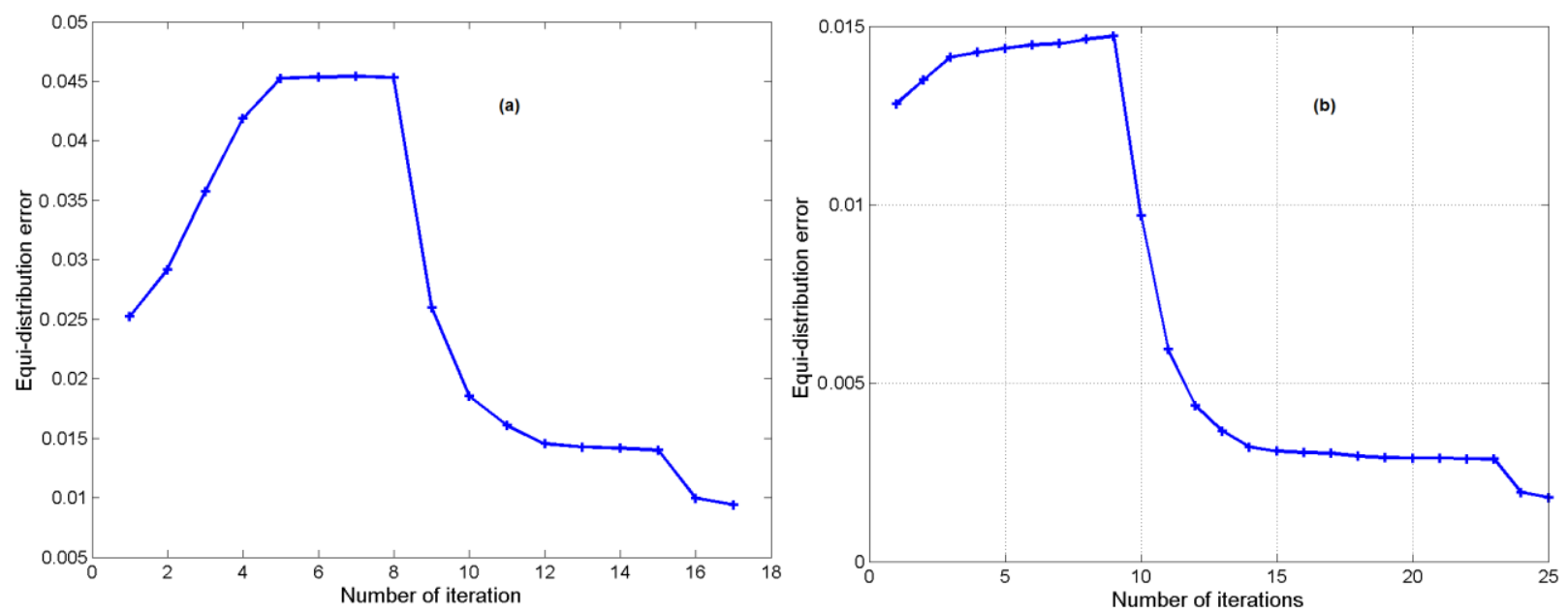

Figure 22: The evolution of volume equi-distribution error with the adaptation iteration. (a) the first example (Figure 18), (b) the second example (Figure 20).

\subsection{Test time-dependent anisotropic distribution}

Two examples were considered for time-dependent mesh optimization using the hybrid adaptive algorithm. Both tests were adopted from Chacon, Delzanno and Finn (2011) and Delzanno et al. (2008). The first one is a traveling cosine wave which represents a solution of a hyperbolic system, ideally the outgoing waves should not reflect back into the computational domain. The dynamic adaptive meshes want to track the time-dependent anisotropic distribution of the solution. The monitor function is given by Eqn. (20) which is a function of time

$f(x, y)=\frac{1}{2.0+\cos \left[8.0 \pi \sqrt{\left(x-0.5-c_{x} t\right)^{2}+\left(y-0.5-c_{y} t\right)^{2}}\right]}$

where the initial center of the cosine wave was at $(0.5,0.5)$ with a constant translating velocity provided by $\left(c_{x}, c_{y}\right)=(-0.5,0.0)$. The goal mesh resolution at time zero was shown in Figure 23. Snapshots of dynamic adaptation of the polygonal meshes at three instants were demonstrated in Figure 24. The top two sub-panels were optimized meshes obtained at time $t=0.0 \mathrm{~s}$ and $t=0.5 \mathrm{~s}$, respectively. The bottom two sub-panels illustrated the optimization of mesh at time $t=0.75 \mathrm{~s}$, while the left side was an intermediate mesh after the coarsening and 
two global mesh regulation sweeps finished and the right side was the final optimized mesh to meet the equi-distribution requirement. This example demonstrates the capability of the hybrid adaptive algorithm for dynamic optimization of polygonal mesh to track the evolution of the underlying physical field. The total number of iterations is around 30, which would be even less if turning off the global mesh regulation process. In fact, the hybrid adaptation does several coarsening and refinement sweeps which can substantially reduce the volume equi-distribution error, which is a typical approach for conventional dynamic AMR in the numerical solution of PDEs.

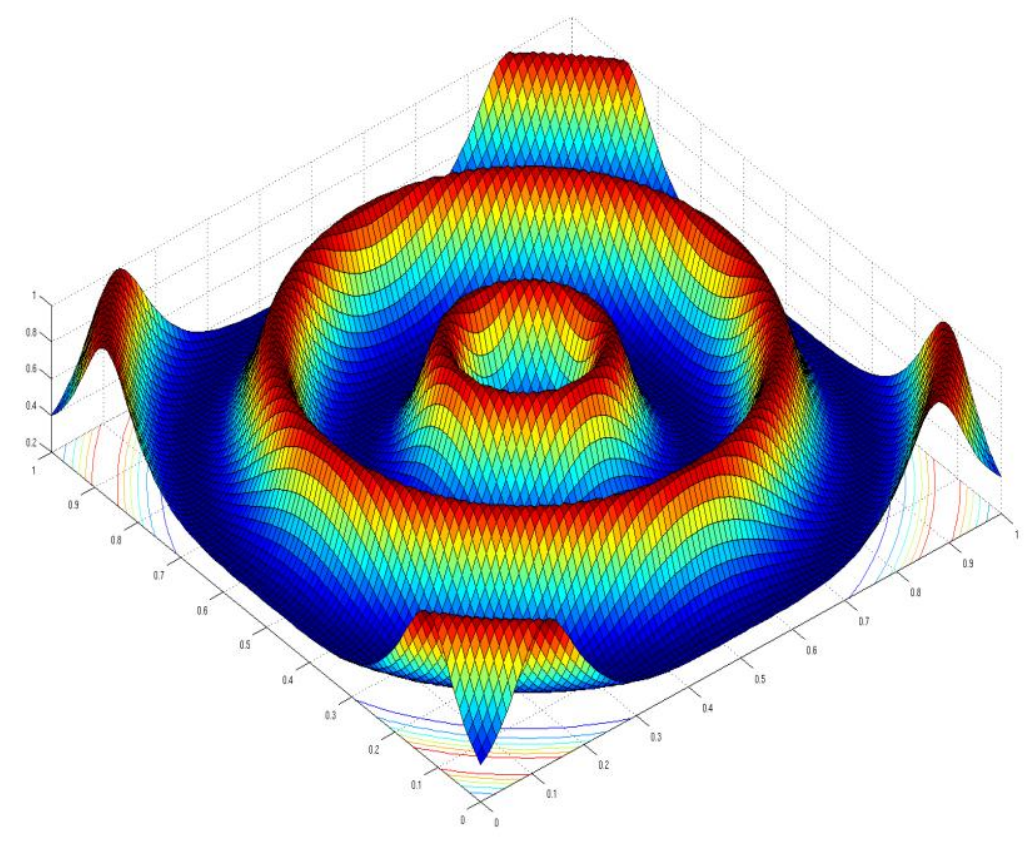

Figure 23: The mesh resolution in a unit square at time zero. The red color illustrates dense mesh (e.g. small mesh size) needed while the blue color stands for region with coarse mesh. 

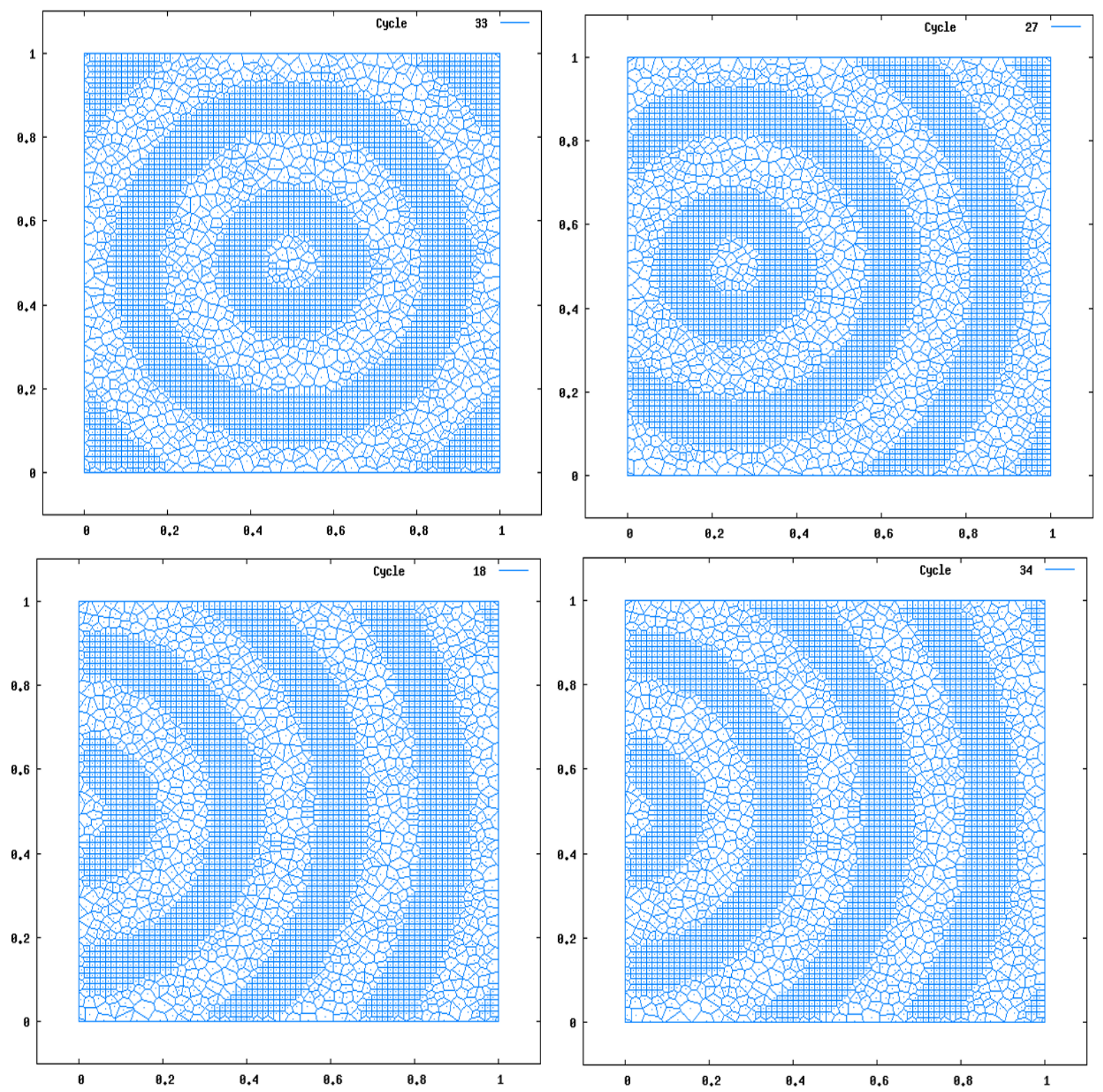

Figure 24: Snapshots of the optimized polygonal meshes obtained by the hybrid adaptive algorithm. Top left: optimized mesh at time $t=0.0 \mathrm{~s}$; Top right: optimized mesh at time $t=0.50 \mathrm{~s}$; Bottom: an intermediate (left panel) and the final optimized (right panel) polygonal mesh at time $t=0.75 \mathrm{~s}$. The bandwidth of mesh resolution was $1.25 \times 10^{-4}$.

The second test case for time-dependent anisotropic meshing is given by Eqn. (21) which represents a rigidly moving body in incompressible flow (Chacon et al. 2011),

$$
f(x, y)=1.0+4.0 \exp \left[-r(x, y)^{2}\left(\frac{\cos ^{2} \theta(x, y, t)}{\sigma_{x}^{2}}+\frac{\sin ^{2} \theta(x, y, t)}{\sigma_{y}^{2}}\right)\right]
$$


where $\sigma_{x}^{2}=0.03, \sigma_{y}^{2}=0.003, \quad r(x, y)=\sqrt{(x-0.5)^{2}+(y-0.5)^{2}}, \quad \theta(x, y, t)=\theta_{0}(x, y)-$ $\Omega_{0} t$, and $\theta_{0}(x, y)=\arctan \left(\frac{y-0.5}{x-0.5}\right)$. The body rigidly rotates in a clockwise direction around the center $(0.5,0.5)$. This challenging problem is intended to test the anisotropic meshing capability of the hybrid adaptive algorithm for Lagrangian-like mesh motion applications. The rotation of a rigid body poses a challenge to mesh generation and optimization due to possible large change of the aspect ratio and the skewness of the mesh which is different from the pure translation in the first problem

The initial physical field is a stationary distribution of a rod in a unit square. The dynamic mesh obtained will track the rotating distribution of the field, which is analogous to an induced flow field by the rotating rod. Figure 25 illustrates the mesh resolution at time zero. The lighter region stands for dense mesh needed due to the concentration of kinetic energy of the physical field. The snapshots of dynamic polygonal meshes obtained from the hybrid adaptive algorithm are shown in Figure 26. The top panel is for the instant $t=0.5 \mathrm{~s}$ while the bottom panel is for the instant $t=1.0 \mathrm{~s}$. Intermediate meshes are also included for comparison to the final optimized meshes at each instant, respectively. It is seen that the optimized polygonal meshes have effectively avoided possible mesh distortion and tangling phenomena that has plagued in other mesh optimization algorithms.

From above two examples it is seen that the novel algorithm proposed here demonstrates its capability to change the underlying topology of the mesh that would offer significant benefit over pure moving mesh methods for arbitrary Lagrangian-Eulerian (ALE) fluid dynamics modeling involving remapping and rezoning. Because the generation of mesh is based on Voronoi tessellations, the topology itself is optimal which therefore can effectively eliminate shape distortion and large aspect ratio issues. As we saw before, the optimized mesh can locally 
adapt to large deformation, shock wave, anisotropic media, and among local feature without addition overhead of computation.

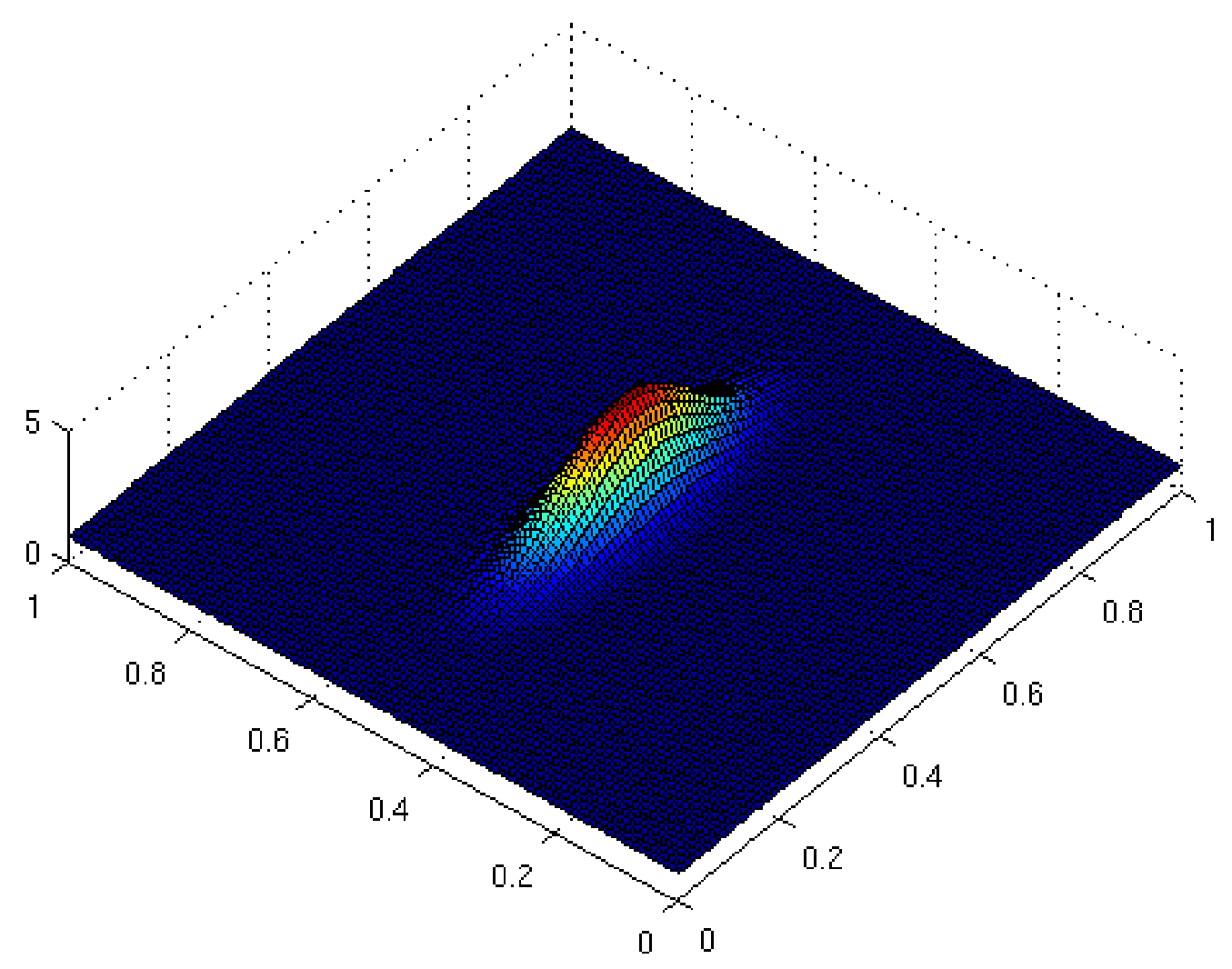

Figure 25: The mesh resolution requirement at time zero for a rotating rod in a stationary field. The region with bright color stands for dense mesh needed while the blue color for coarse mesh distribution in a unit square. 

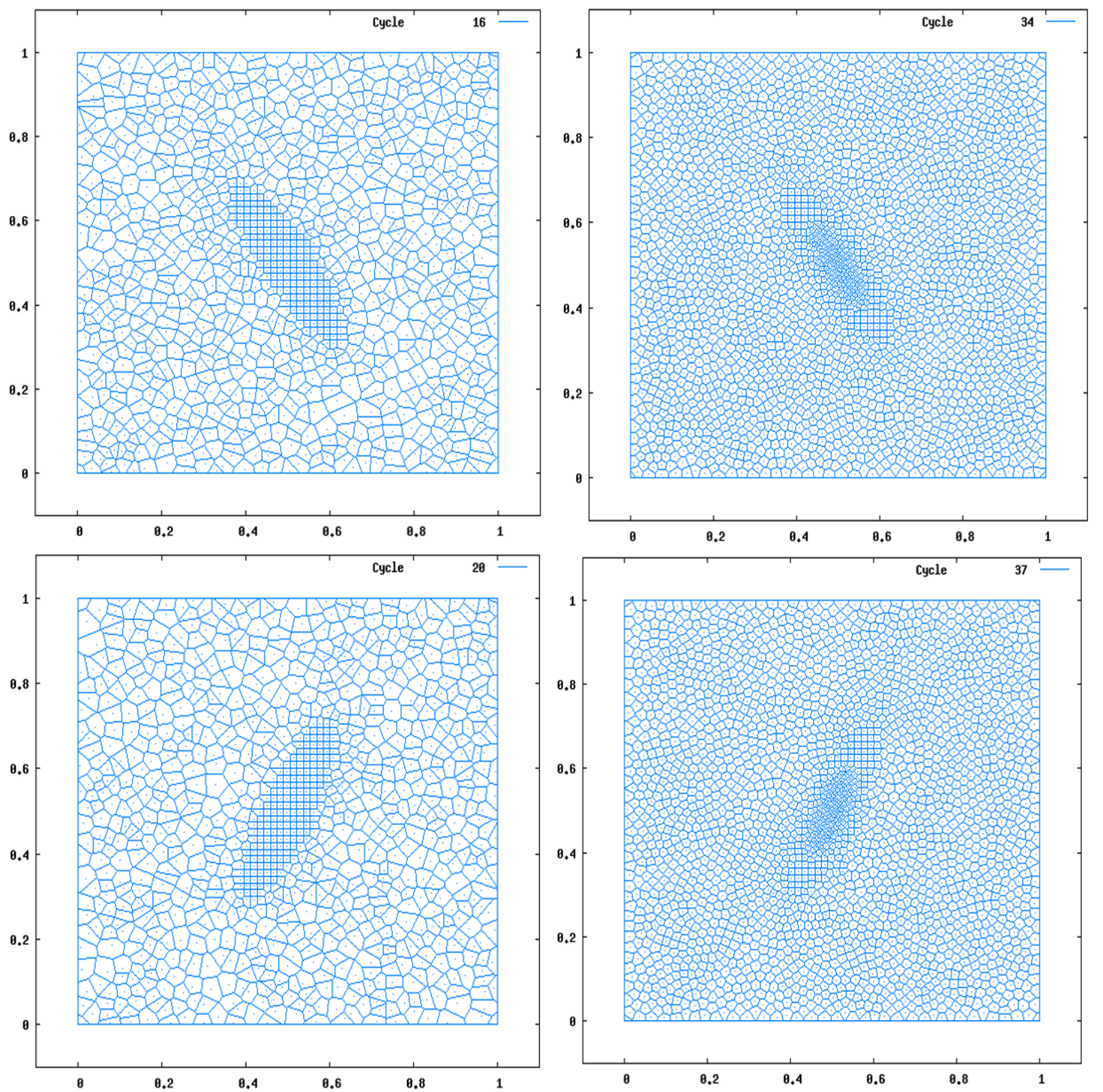

Figure 26: The optimal polygonal meshed obtained at time $t=0.50 \mathrm{~s}$ (top panel) and $t=1.0 \mathrm{~s}$ (bottom panel) for the rigid body rotation in a unit square. The left side of each panel represents an intermediate mesh to compare to the right side (the final optimized mesh). The bandwidth of mesh resolution was $1.25 \times 10^{-4}$.

\section{Conclusion}

We have presented a novel hybrid $h$-adaptive algorithm for polygonal mesh optimization in 2D based on Voronoi tessellations and its topological dual, the Delaunay triangulations and the 
equi-distribution principle. The complete implementation has been established including internal and boundary node process. The major components of the novel approach include coarsening, refinement, local smoothing and global mesh regulation. The algorithm has also provided options for using global mesh regulation between the coarsening and the refinement, the so-called synchronous or asynchronous link. Given a monitor function (i.e., density function) the mesh will converge to an optimal shape polygonal mesh that meets the equi-distribution of the monitor function. Numerical experiments have demonstrated a faster convergence rate than pure $r$ adaptive approach such as CVT r-adaptive and the so-called Lloyd's iterations. Finally, The hybrid $h$-adaptive can be extended to 3D problems, and from convex to non-convex geometries through domain decomposition.

Finally, in Part II: Applications, a separate paper, the new algorithm will be tested on a variety of $2 \mathrm{D}$ examples including the adaptive solution of elliptic PDE problems for its robustness, efficiency and effectiveness. The discretization will be based on finite volume methodology. Other engineering applications of the hybrid $h$-adaptive algorithm such as elastic body and multiphase flow will be discussed with detailed comparison of convergence rate, the equi-distribution numerical errors and CPU time for individual sweeps. The efficiency comparison between synchronous and asynchronous approaches will also be demonstrated.

\section{Acknowledgments}

The author is very grateful to Dr. M.J. Shashkov for his support and instruction. The numerical code to generate Voronoi tessellations was provided by Dr. Shashkov and Dr. R. Loubere. The author has received lots of support from and has useful discussions with Dr. P. Swart, Dr. K. Rasmussen and Dr. R. Garimella at the Applied Mathematics and Plasma Science Group, LANL. 
This work was partially funded by US Department of Energy Advanced Simulation and Computing (ASC) program, under the auspices of the US Department of Energy by Los Alamos National Laboratory. The author would like to thank anonymous reviewers for their time and valuable comments which help us to clarify the confusion and correct errors during the preparation.

\section{References}

1. Bank, R.E. and R.K. Smith, Mesh smoothing using a posteriori error estimates, SIAM J. Numer. Anal. 34(3):979-997, 1997

2. Barth, T.J. and D.C. Jespersen, The design and application of upwind schemes on unstructured meshes, $27^{\text {th }}$ AIAA Aerospace Sciences Meeting, Paper No. AIAA-89-0366, Jan 9-12, 1989

3. Brackbill, J.U. and J.S. Saltzman, Adaptive zoning for singular problems in two dimensions, J. Comput. Phys., 46(3):342-368, 1982

4. Budd, C. and J.W. Williams, Parabolic Monge-Ampere methods for blow-up problems in several spatial dimensions, J. Phys. A, 39:5425-5444, 2006

5. Budd, C.J. and J.F. Williams, Moving mesh generation using the parabolic Monge-Ampere equation, SIAM J. Sci. Comput., 31(5):3438-3465, 2009

6. Cappellari, M. and Y. Copin, Adaptive spatial binning of integral-field spectroscopic data using Voronoi tessellations, Mon. Not. R. Astron. Soc., 342:345-354, 2003

7. Carpenter, V.J.G., Adaption of unstructured meshes using node movement. $5^{\text {th }}$ Inter. Conference on Numerical Grid Generation in Computational Field Simulations, Soni, B.K., Thompson, J.F. Hauser, J. and P. Eiseman (ed.), 269-278, 1996 
8. Chacon, L., Delzanno, G.L. and J.M. Finn, Rboust, multidimensional mesh-motion based on Monge-Kantorovich equidistribution, J. Comput. Phys., 230:87-103, 2011

9. Delzanno, G.L. and J.M. Finn, The fluid dynamic approach to equidistribution methods for grid adaptation, Comput. Phys. Commun. 182:330-346, 2011

10. Delzanno, G.L., Chacon, L., Finn, J.M., Chung, Y. and G. Lapenta, An optimal robust equidistribution method for two-dimensional grid adaptation based on Monge-Kantorovich optimization, J. Comput. Phys. 227:9841-9864, 2008

11. Deville, M.O., Fischer, P.F. and E.H. Mund, High-order Methods for Incompressible Fluid Flow, Cambridge University Press, 2002

12. Dorfler, W., A convergent adaptive algorithm for Poisson's equation, SIAM J. Numer. Anal., 33(3): 1106-1124, 1996

13. Du, Q. and M. Gunzburger, Grid generation and optimization based on centroidal Voronoi tessellations, Appl. Math. Comput., 133:591-607, 2002

14. Du, Q., Faber, V. and M. Gunzburger, Centroidal Voronoi tessellations: applications and algorithms, SIAM Review, 41(4):637-676, 1999

15. Du, Q., Gunzburger and L. Ju, Advances in studies and applications of centroidal Voronoi tessellations, Numer. Math. Theor. Meth. Appl., 3(2):119-142, 2010

16. Emeliamenko, M., Fast multilevel CVT-based adaptive data visualization algorithm, Numer. Math. Theor. Meth. Appl., 3(2):195-211

17. Evazi, M. and H. Mahani, Generation of Voronoi grid based on vorticity for coarse-scale modeling of flow in heterogenous formations, Transp. Porous Media, 83:541-572, 2010 
18. Feng, Z.G., Michaelides, E.E. and S. Mao, On the drag force of a viscous sphere with interfacial slip at small but finite Reynolds numbers, Fluid Dynamics Research, 44(2), 025502, 2012

19. Ferziger, J.H. and M. Peric, Computational Methods for Fluid Dynamics, Springer 2002

20. Garimella, R., Kucharik, M. and M. Shashkov, An effective linearity and bound preserving conservative interpolation (remapping) on polyhedral meshes, Computers and Fluids, 36(2):224-237, 2007

21. Gersho, A., Asymptotically optimal block quantization, IEEE Trans Infor Theory, 25(4):373380,1979

22. Huang, W. and W. Sun, Variational mesh adaptation: error estimates and monitor functions, J. Comput. Phys., 184:619-648, 2003

23. Huang, W., Metric tensors for anisotropic mesh generation, J. Comput. Phys., 204:633-665, 2005

24. Jones, P.W., First- and second-order conservative remapping schemes for grids in spherical coordinates, Mon. Weather Rev., 127(9):2204-2210, 1999

25. Ju, L., Gunzburger, M. and W. Zhao, Adaptive finite element methods for elliptic PDEs based on conforming centroidal Voronoi-Delaunay triangulations, SIAM J. Sci. Comput., 28(6):2023-2053, 2006

26. Khamayseh, A., Almedida, V. de and G. Hansen, Hybrid surface mesh adaptation for climate modeling, Numer. Math. Theor. Meth. Appl., 1(4):410-434, 2008

27. Kim, D.-S. (ed.), The $2^{\text {nd }}$ International Symposium on Voronoi Diagrams in Science and Engineering, Oct. 10-13, 2005, Seoul, Korea 
28. Knupp, P. and S. Steinberg, Fundamentals of Grid Generation, CRC Press, Boca Raton, FL, 1994

29. Kucharik, M., Shashkov, M. and B. Wendroff, An efficient linearity-and-bound-preserving remapping methods, J. Comput. Phys., 188:462-471, 2003

30. Lapenta, G., Variational grid adaptation based on the minimization of local truncation error: time-independent problems, J. Comput. Phys., 193:159-179, 2003

31. Li, S., Petzold, L. and Y. Ren, Stability of moving mesh systems of partial differential equations, SIAM J. Sci. Comput., 20(2):719-738, 1998

32. Lipnikov, K. and M. Shashkov, The error-minimzation-based strategy for moving mesh methods, Commun. Comput. Phys., 1(1):53-81, 2006

33. Liseikin, V.D., Grid Generation Methods, Springer, Berlin, New York, 1999

34. Lloyd, S., Least squares quantization in PCM, IEEE Trans. Infor. Theory, 28:129-137, 1982

35. Loubere, R., Maire, P.H., Shashkov, M., Breil, J. and S. Galera, ReALE: a reconnectionbased arbitrary Lagrangian Eulerian method, J. Comput. Phys., 229:4724-4761, 2010

36. Ma, Z., Chen, H. and C. Zhou, A study of point moving adaptivity in gridless method, Comput. Methods Appl. Mech. Engrg., 197:1926-1937, 2008

37. Mao, S. and I.B. Celik, Modeling of indoor airflow and dispersion of aerosols using immersed boundary and random flow generation methods, Computers and Fluids, 39:12751283,2010

38. Mao, S., Feng, Z.G. and E.E. Michaelides, Large-eddy simulation of low-level jet-like flow in canopy, Environmental Fluid Mechanics, 7(1):73-93, 2007 
39. Mao, S., Leclerc, M.Y. and E.E. Michaelides, Passive scalar flux footprint analysis over horizontally inhomogeneous plant canopy using large-eddy simulation, Atmospheric Environment, 42(21):5446-5458, 2008

40. Margolin, L.G. and M. Shashkov, Second-order sign-preserving remapping on general grids, LA-UR-02-525, Los Alamos National Laboratory (also J. Comput. Phys., 184(1): 266-298, 2003), 2002

41. Menon, S. and D.P. Schmidt, Conservative interpolation on unstructured polyhedral meshes: An extension of the supermesh approach to cell-centered finite volume variables, Comput. Methods Appl. Mech, Engrg., 200:2797-2804, 2011

42. Noh, W.F., Errors for calculations of strong shocks using artificial viscosity and artificial heat flux, J. Comput. Phys., 72:78-120, 1987

43. Norris, S.E., Were, C.J., Richards, P.J. and G.D. Mallinson, A Voronoi-based ALE solver for the calculation of incompressible flow on deforming unstructured meshes, Int. J. Numer. Meth. Fluids, DOI:10.1002/fld.2234, 2010

44. Okabe, A., Boots, B., Sugihara, K. and S.N. Chiu, Spatial Tessellations: Concepts and Applications of Voronoi Diagrams, $2^{\text {nd }}$ Edition, Wiley, Chichester, 2000

45. Prosperetti, A. and G. Tryggvason, Computational Methods for Multiphase flow, Cambridge University Press, 2007

46. Ringler, T., Ju, L. and M. Gunzburger, A multiresolution method for climate system modeling: application of spherical centroidal Voronoi tessellations, Ocean Dynamics, $58: 475-498,2008$

47. Shewchuk, J.R., Lecture Notes on Delaunay Mesh Generation, Department of Electric Engineering and Computer Science, University of California, Berkeley, 1999 
48. Shyy, W., Udaykumar, H.S., Rao, M.M. and R.W. Smith, Computational Fluid Dynamics with Moving Boundaries, Taylor \& Francis, 1996 (Dover 2007).

49. Springel, V., E pur si muove: Galiliean-invariant cosmological hydrodynamical simulations on a moving mesh, arXiv:0901.4107v3, Mon. Not. R. Astron Soc., 1-67, 2009

50. Stein, K., Tezduyar, T. and R. Benney, Mesh moving techniques for fluid-structure interactions with large displacements, ASME J. Applied Mechanics, 70:58-63, 2003

51. Tabarrael, A. and N. Sukumar, Adaptive computations using material forces and residualbased error estimators on a quadtree meshes, Comput. Methods Appl. Mech. Engrg., 196:2657-2680, 2007

52. Taniguchi, N. and T. Kobayashi, Finite volume method on the unstructured grid system, Computers and Fluids, 19:287-295, 1991

53. Thompson, J.F., Soni, B.K. and N.P. Weatherill, Handbook of Grid Generation, CRC Press, Boca Baton, FL

54. Thompson, J.F., Warsi, Z.U.A. and C.W. Mastin, Numerical Grid Generation: Foundations and Applications, North-Holland, New York, 1985

55. Vachal, P. and P.-H. Maire, Discretizations for weigted condition number smoothing on general unstructured meshes, Computers and Fluids, 46:479-485, 2011

56. Weller, H., Weller, H.G. and A. Fournier, Voronoi, Delaunay, and block-structured mesh refinement for solution of the shallow-water equations on the sphere, Monthly Weather Review, 137:4208-4224, 2009

57. Winslow, A., Adaptive mesh zoning by the equipotential method, Technical Report UCID19062, Lawrence Livermore Laboratory, 1981 
58. Zienkiewicz, O.C. and J.Z. Zhu, A simple error estimator and adaptive procedure for practical engineering analysis, Int. J. Numer. Methods Eng., 24:337-357, 1987

Significance and novelty:

- A new hybrid $h$-adaptive mesh algorithm with the capability to variable mesh topology

- The algorithm is based on equi-distribution principle and Voronoi tessellations

- The coarsening and refinement processes can be asynchronous or synchronous

- A new double thresholds marking strategy and a local smoothing have been introduced

- The hybrid algorithm can converge to arbitrary mesh resolution 